\title{
SPECTRAL ENERGY DISTRIBUTION ANALYSIS OF CLASS I AND CLASS II FU ORIONIS STARS
}

\author{
Luciana V. Gramajo ${ }^{1}$, Javier A. Rodón ${ }^{2}$, and Mercedes Gómez ${ }^{1}$ \\ ${ }^{1}$ Observatorio Astronómico, Universidad Nacional de Córdoba, Argentina, Laprida 854, 5000 Córdoba, Argentina; \\ luciana@oac.uncor.edu,mercedes@oac.uncor.edu \\ ${ }^{2}$ European Southern Observatory, Alonso de Córdova 3107, Vitacura, Casilla 19001, Santiago 19, Chile; jrodon@eso.org \\ Received 2013 June 4; accepted 2014 February 11; published 2014 May 6
}

\begin{abstract}
FU Orionis stars (FUors) are eruptive pre-main sequence objects thought to represent quasi-periodic or recurring stages of enhanced accretion during the low-mass star-forming process. We characterize the sample of known and candidate FUors in a homogeneous and consistent way, deriving stellar and circumstellar parameters for each object. We emphasize the analysis in those parameters that are supposed to vary during the FUor stage. We modeled the spectral energy distributions of 24 of the 26 currently known FUors, using the radiative transfer code of Whitney et al. We compare our models with those obtained by Robitaille et al. for Taurus class II and I sources in quiescence periods by calculating the cumulative distribution of the different parameters. FUors have more massive disks: we find that $\sim 80 \%$ of the disks in FUors are more massive than any Taurus class II and I sources in the sample. Median values for the disk mass accretion rates are $\sim 10^{-7} M_{\odot} \mathrm{yr}^{-1}$ versus $\sim 10^{-5} M_{\odot} \mathrm{yr}^{-1}$ for standard young stellar objects (YSOs) and FUors, respectively. While the distributions of envelope mass accretion rates for class I FUors and standard class I objects are similar, FUors, on average, have higher envelope mass accretion rates than standard class II and class I sources. Most FUors $(\sim 70 \%)$ have envelope mass accretion rates above $10^{-7} M_{\odot} \mathrm{yr}^{-1}$. In contrast, $60 \%$ of the classical YSO sample has an accretion rate below this value. Our results support the current scenario in which changes experimented by the circumstellar disk explain the observed properties of these stars. However, the increase in the disk mass accretion rate is smaller than theoretically predicted, although in good agreement with previous determinations.
\end{abstract}

Key words: circumstellar matter - infrared: stars - stars: formation - stars: pre-main sequence - stars: variables: $\mathrm{T}$ Tauri, Herbig Ae/Be

Online-only material: color figures, figure set, machine-readable and VO tables

\section{INTRODUCTION}

FU Orionis stars (FUors) are a class of variable young stellar objects (YSOs) that show brightness variations of the eruptive type (Herbig 1977). The main feature observed in these variables is a sudden increase in brightness (3-6 mag in the optical), in an elapse time of a few months. This episode is known as the "outburst," after which the object remains bright for years or decades and then fades, in a few centuries, back to the preoutburst stage. The outburst, however, occurs in a different way for each FUor (see, e.g., Hartmann \& Kenyon 1996; Clarke et al. 2005a).

These stars exhibit several indicators of youth, such as the presence of the lithium $6707 \AA$ line in optical spectra, and the association with reflection nebulae and infrared excesses originating from dust grains in circumstellar disks. Moreover, they are spatially and kinematically related to known starforming regions, and in some cases FUors have high extinction values in the optical, suggesting that they are still embedded in the parent cloud material (see, e.g., Hartmann \& Kenyon 1996).

FUors show several properties that strongly suggest the presence of a circumstellar disk, such as broad spectral energy distributions (SEDs; Kenyon et al. 1988), stellar spectral types that become progressively colder with increasing wavelength (Hartmann \& Kenyon 1985; Kenyon et al. 1988), spectral line widths that increase with decreasing wavelength (Hartmann \& Kenyon 1987a, 1987b), double-peaked line profiles in highresolution optical and near-infrared (NIR) spectra (Hartmann \& Kenyon 1985; Kenyon et al. 1988) as well as P-Cygni profiles with no evidence for redshifted emission or absorption
(Kenyon et al. 1988; Hartmann \& Calvet 1995), and finally, deep, broadened infrared CO-bands in absorption (Kenyon \& Hartmann 1988; Reipurth \& Aspin 1997).

Another class of eruptive variables is the so-called EXOR stars, named after EX Lup, the progenitor of the class (Herbig 1989, 2008). Their optical brightness increases by $1-4$ mag on time scales of weeks or months, then fades back during a few months to its original state. During the low activity stage they exhibit T Tauri-like characteristics, whereas during the outburst stage they usually display emission in the optical spectrum as well as in the infrared CO-bandheads (e.g., Aspin et al. 2010).

To reproduce the SEDs of FUors, modelers use dusty disks and infalling envelopes (e.g., Hartmann \& Kenyon 1985; Kenyon et al. 1988; Calvet et al. 1991; Hartmann \& Calvet 1995; Calvet 1998; Whitney et al. 2003a, 2003b). Indeed the presence of a circumstellar disk is essential to explain the FU Orionis phenomenon. The disk is where material coming from the surrounding infalling envelope accumulates, heats-up, and finally destabilizes the structure of the disk itself, causing a thermal (Frank et al. 1992; Bell \& Lin 1994) and/or a gravitational (Zhu et al. 2009, 2010; Vorobyov \& Basu 2005, 2006, 2010) instability that eventually leads to the characteristic outburst.

During this episode an increase of the brightness takes place, affecting mainly the optical wavelengths since the excess emission comes from the inner regions of the disk, which are heated by the viscous dissipation released after the instability has triggered an increase in the disk accretion rate. Frank et al. (1992) suggests that the central objects of the FUor systems alternate between low $\left(10^{-7} M_{\odot} \mathrm{yr}^{-1}\right)$ and high $\left(10^{-4} M_{\odot} \mathrm{yr}^{-1}\right)$ mass accretion rates. The former corresponds to a low activity, 
quiescent state, whereas the latter corresponds to periods of high activity. Furthermore, the transformations undergone by the disk are what cause the observed phenomenon. Similarly, EXOR events are also attributed to thermal instabilities in the inner disks (Aspin 2011b).

Currently, 26 FUors have been identified and classified as class I or class II objects according to the shape of their SEDs (Lada 1987). This includes the "confirmed" FUors, for which the sudden increase in brightness has been recorded, and the "candidate" FUors, which share many, if not all, of the properties of bona-fide FUors but for which an outburst has not been observed. In this paper we model and analyze the SEDs of 24 confirmed and candidate FUors to determine the physical and geometrical parameters of the star and the disk.

In Section 2 we present the sample, describe the adopted model and outline the procedure used in the SED modeling. In Section 3 we analyze the individual sources. Our results are presented in Section 4. Finally, in Section 5 we summarize the results and conclusions.

\section{RADIATIVE TRANSFER SED MODELING}

\subsection{The Sample}

Our sample includes 24 of the 26 known and candidate class I and II FU Orionis stars. The FUors Ca I 136, which is in NGC 3372 in the Carina nebula (Tapia et al. 2006), and V733 Cep, which is in the dark cloud L1216 (Reipurth et al. 2007; Peneva et al. 2010), are both identified as FUor candidates, but were not included as not enough fluxes to construct the SED were available in the literature. In particular, for these objects only fluxes in the near-infrared wavelengths have been published.

For some of the 24 remaining objects, only fluxes in the optical, NIR, and mid-infrared (MIR) wavelengths are available. The lack of measurements in the far-infrared (FIR) and submillimeter (sub-mm) spectral regions makes it only possible to derive reliable parameters for the protostar and the inner disk, since the outer regions of the disk and the envelope emit mostly at FIR and sub-mm wavelengths.

Table 1 presents our sample and summarizes the main properties of each source, such as luminosity, optical extinction $\left(A_{\mathrm{V}}\right)$, variation in the $K$-band $(\Delta \mathrm{K})$, year of the outburst (if registered), association with molecular outflows/jets, SED class, spectral type, distance, and whether the central star is a binary.

We classified the sources according to their observational properties. In particular, we used the $\mathrm{CO}$ band at $2.3 \mu \mathrm{m}$ to classify the objects as confirmed FUors if it appears in absorption (e.g., Reipurth \& Aspin 1997; Hartmann et al. 2004), or as a FUor candidate otherwise (i.e., if the band is in emission or absent, e.g., Reipurth \& Aspin 2004a; Aspin 2011b). The FUors in our sample were subsequently classified as class II visible pre-main sequence stars or as embedded class I objects. Sources with $\dot{M}<10^{-7} M_{\odot} \mathrm{yr}^{-1}$ are class II stars surrounded by disks, whereas objects with $\dot{M}>10^{-7} M_{\odot} \mathrm{yr}^{-1}$ are class I objects embedded in infalling envelopes. This criterion is based on the best-fit values for the envelope mass accretion rates listed in Table 1 from Robitaille et al. (2007).

To construct the SED of each object, we compiled all the fluxes available in the literature between $\sim 0.3 \mu \mathrm{m}$ and $\sim 3 \mathrm{~mm}$. Table 2 lists the fluxes compiled for each object. The uncertainties for the fluxes were not always provided in the literature. In those cases we adopted "typical" errors. In addition, we include the MIR spectra published by Green et al. (2006) and Quanz et al. (2007b), when available.

The spectra published by Green et al. (2006) for the sources FU Ori, V1515 Cyg, V1057 Cyg, BBW 76, and V346 Nor were obtained with the Spitzer Infrared Spectrograph (IRS) in the 5-36 $\mu \mathrm{m}$ range in 2003 December and 2004 May. Quanz et al. (2007b) published data obtained with both the Spitzer and the Infrared Space Observatory (ISO) telescopes. In this work we include the Spitzer-IRS spectra for RNO 1B, RNO 1C, Par 21, and L1551 IRS5, which were observed in the 5-14 $\mu \mathrm{m}$ range between 2003 December and 2004 March. The source Re 50 N IRS1 was the only case for which we used spectrum obtained with ISO short wavelength spectrometer (SWS) in the $5-15 \mu \mathrm{m}$ range, which was obtained in 2007 October. We have not included all of the $I S O$ spectra because their signal to noise ratio is low for most of the targets.

\subsection{Procedure}

To analyze the SEDs we used the code developed by Whitney et al. (2003a, 2003b) and the grid of models computed by Robitaille et al. (2006). Briefly, the dust radiative transfer model of Whitney et al. (2003a) reproduces a complete protostellar system comprised by a central source emitting photons and a circumstellar disk and envelope. The code uses a Monte Carlo radiative transfer scheme that follows photon packets emitted by the central star as they are scattered, absorbed, and re-emitted throughout the disk and envelope.

The geometry of the protostellar system (star+disk+infalling envelope) is highly parameterized. The model has 15 modifiable parameters that can be divided into three groups. The central source parameters, that is, stellar mass $\left(M_{*}\right)$, stellar radius $\left(R_{*}\right)$, and stellar temperature $\left(T_{*}\right)$; the infalling envelope parameters comprising the envelope mass accretion rate $(\dot{M})$, envelope outer radius $\left(R_{\max }\right)$, cavity density $\left(\rho_{\text {cav }}\right)$, cavity opening angle $(\theta)^{3}$; and the disk parameters disk mass $\left(M_{\text {disk }}\right)$, disk outeror centrifugal radius $\left(R_{\mathrm{c}}\right),{ }^{4}$ disk inner radius $\left(R_{\min }\right)$, disk mass accretion rate $\left(\dot{M}_{\text {disk }}\right)$, disk radial density exponent (A), disk scale height exponent (B), and disk fiducial scale height $\left(z_{01}\right) .^{5}$ The ambient density surrounding the young stars $\left(\rho_{\mathrm{amb}}\right)$ is included as the 15th parameter. In addition to these parameters, the inclination angle to the line of sight $(i)$ is one of the most critical parameters when modeling the SEDs. In our analysis we adopted a value of $z_{01}=0.03 R_{*}$, as it remains practically unchanged for this type of objects.

We used the Robitaille et al. (2006) grid (also called "the SED fitting tool") of YSO models ${ }^{6}$ to obtain an initial model for each source. Robitaille et al. (2006) used the Whitney et al. (2003b) code to compute a grid of 20,000 axisymmetric radiation transfer models of YSOs at 10 viewing angles, resulting in a total of 200,000 SEDs in the wavelength range $0.36-1000 \mu \mathrm{m}$ that cover the "typical" range of values of physical and geometrical parameters that characterize YSOs. The SED fitting tool offers the advantage that data in a wide range of wavelengths can be used simultaneously, without losing information. In addition, this tool allows for the evaluation of the uniqueness or the

\footnotetext{
$3 \theta$ is measured from the axis of rotation at the outer radius of the envelope.

4 The centrifugal radius and the outer-disk radius are usually related. The envelope material falls to $R_{\mathrm{c}}$ at the disk equatorial plane, providing an indication of the extension of the disk (see Whitney et al. 2003b; Robitaille et al. 2006).

5 The disk fiducial scale height, $z_{01}$, is the scale height of the inner disk at $R_{*}$ in units of $R_{*}$.

6 The grid is available at http://caravan.astro.wisc.edu/protostars.
} 
Table 1

The FU Orionis Sample

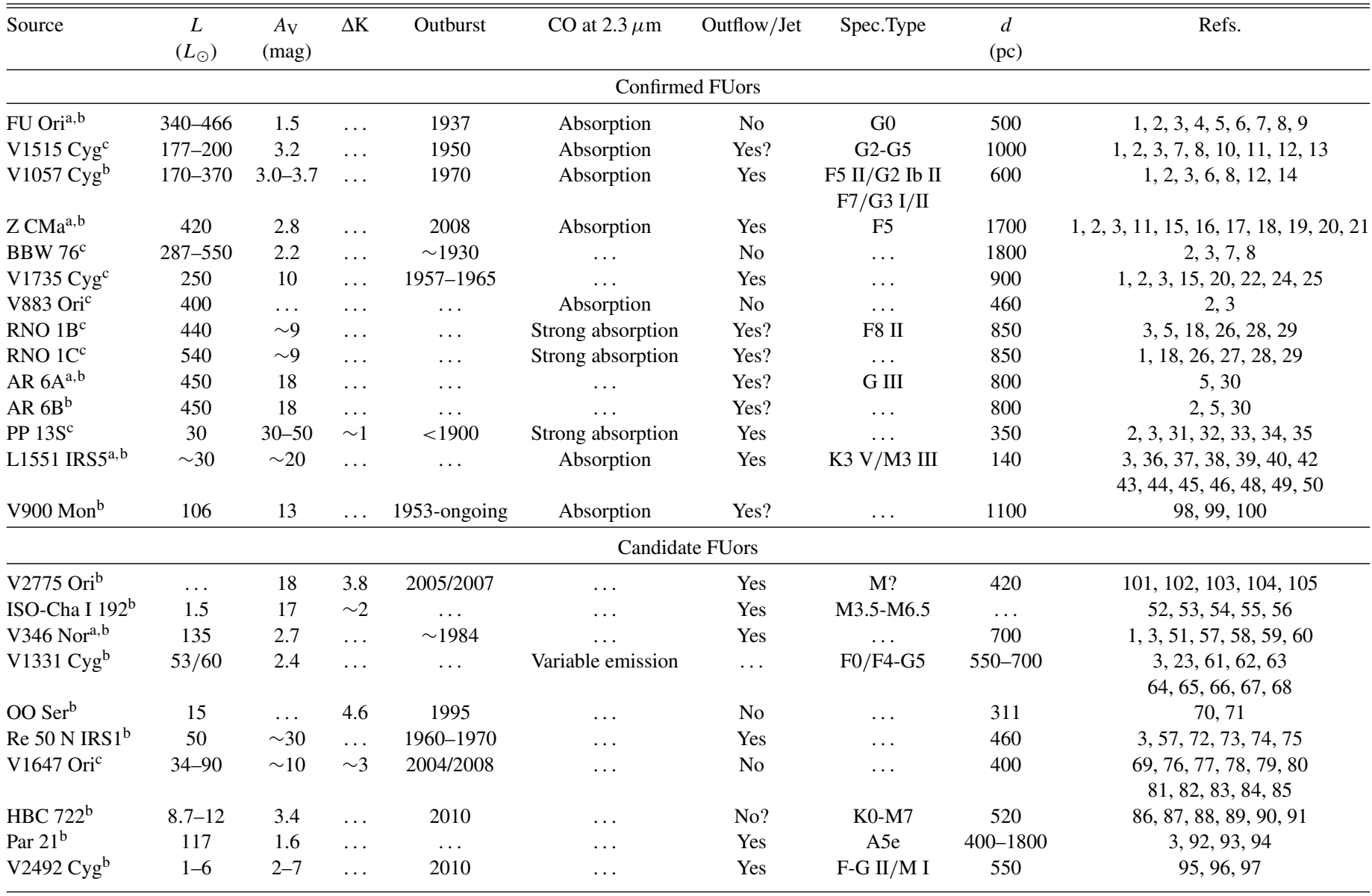

Notes. ${ }^{a}$ Binary star; ${ }^{\mathrm{b}}$ source classified as a class I YSO; ${ }^{\mathrm{c}}$ source classified as a class II YSO.

References. (1) Evans et al. 1994; (2) Hartmann \& Kenyon 1996; (3) Sandell \& Weintraub 2001; (4) Kenyon et al. 2000; (5) Aspin \& Reipurth 2003; (6) Malbet et al. 2005; (7) Green et al. 2006; (8) Zhu et al. 2008; (9) Kenyon et al. 2000; (10) Goodrich 1987; (11) Terranegra et al. 1994; (12) Herbig 1977; (13) Kolotilov \& Petrov 1983; (14) Herbig \& Dahm 2006; (15) Lorenzetti et al. 2001; (16) Herbst et al. 1978; (17) Quanz et al. 2006; (18) Polomski et al. 2005; (19) Kenyon et al. 1989; (20) Grankin \& Artemenko 2009 (21) Hartmann et al. 1989 (22) Sato et al. 1992; (23) Levreault 1988; (24) Connelley et al. 2007; (25) Harvey et al. 2008; (26) Staude \& Neckel 1991; (27) Kenyon et al. 1993b; (28) McMuldroch et al. 1995; (29) Greene \& Lada 1996; (30) Moriarty-Schieven et al. 2008; (31) Cohen \& Schwartz 1983; (32) Tapia et al. 1997; (33) Sandell \& Aspin 1998; (34) Aspin \& Reipurth 2000; (35) Aspin \& Sandell 2001; (36) Strom et al. 1976; (37) Snell et al. 1980; (38) Cohen et al. 1984; (39) Snell et al. 1985; (40) Doppmann et al. 2005; (41) Prato et al. 2009; (42) Mundt et al. 1985; (43) Carr et al. 1987; (44) Adams et al. 1987; (45) Carr 1990; (46) Davis et al. 1995; (47) Devine et al. 1999; (48) Rodríguez et al. 2003; (49) Osorio et al. 2003; (50) Rodríguez et al. 1998; (51) Pfalzner 2008; (52) Mattila et al. 1989; (53) Persi et al. 1999; (54) Gómez \& Mardones 2003; (55) Gómez et al. 2004; (56) Persi et al. 2007; (57) Strom \& Strom 1993; (58) Prusti et al. 1993; (59) Gredel 1994; (60) Chavarria 1981; (61) Reipurth et al. 1997; (62) Carr 1989; (63) McMuldroch et al. 1993; (64) Biscaya et al. 1997; (65) Mundt \& Eislöffel 1998; (66) Henning et al. 1998; (67) Lorenzetti et al. 2000; (68) Hamann \& Persson 1992; (69) Ábrahám et al. 2004b; (70) de Lara et al. 1991; (71) Hodapp et al. 1996; (72) Heyer et al. 1990; (73) Reipurth \& Aspin 1997; (74) Stanke et al. 2000; (75) Lee et al. 2002; (76) Andrews et al. 2004; (77) Briceño et al. 2004; (78) McGehee et al. 2004; (79) Reipurth \& Aspin 2004b; (80) Aspin 2011a; (81) Vacca et al. 2004; (82) Walter et al. 2004; (83) Muzerolle et al. 2005; (84) Acosta-Pulido et al. 2007; (85) Lis et al. 1999; (86) Miller et al. 2011; (87) Semkov et al. 2010; (88) Laugalys et al. 2006; (89) Cohen \& Kuhi 1979; (90) Green et al. 2011; (91) Dunham et al. 2012; (92) Allen et al. 2004; (93) Liu et al. 2011; (94) Staude \& Neckel 1992; (95) Covey et al. 2011; (96) Straizys et al. 1989; (97) Aspin 2011b; (98) Reipurth et al. 2012 (99) Gregorio-Hetem 2008; (100) Lombardi et al. 2011; (101) Caratti o Garatti et al. 2011; (102) Fischer et al. 2012; (103) Sandstrom et al. 2007; (104) Menten et al. 2007; (105) Kim et al. 2008.

goodness of the fit, calculating the $\chi^{2}$-per-data point value of each model in the grid, following Equation (6) in Robitaille et al. (2007).

For each object analyzed, we selected the best model from the grid of Robitaille et al. (2006) corresponding to the minimum value of $\chi^{2}$ that reproduces the SED, and at the same time, gives reasonable values for the known parameters according to previous determinations from the literature. In other words for each source we selected the model with the smallest $\chi^{2}$ that provides parameter values in agreement with those already published. Spectra were not used in this procedure. However, in all cases the selected models reproduce the spectra well enough. These initial models were used as the starting points for a detailed analysis with the Whitney et al. (2003a) code. At this step we included in the modeling the available MIR spectra. The direct application of the Whitney et al. (2003a) code allows, among other things, the introduction of discrete step variations in the values of the parameters. In this way, a refined model (i.e., a fit with a better $\chi^{2}$ can be obtained), since in the Robitaille et al. (2006) grid step variations are fixed.

As mentioned before, the sample we analyze consists of class I and class II objects. The parameters for which a more accurate value can be obtained from the SED modeling are related to the envelope in the first case, and to the disk in the second. 
Table 2

Fluxes Used to Construct the SED of Each Source

\begin{tabular}{lcc}
\hline \hline $\begin{array}{l}\lambda \\
(\mu \mathrm{m})\end{array}$ & $\begin{array}{c}F_{v} \\
(\mathrm{mJy})\end{array}$ & Ref \\
\hline \multicolumn{3}{c}{ V1057 Cyg } \\
\hline 0.36 & 20 & 73 \\
0.36 & 0.5 & 33 \\
0.44 & 10 & 33 \\
440 & 270 & 73 \\
0.55 & 0.23 & 49 \\
0.55 & 30 & 33 \\
0.55 & 530 & 73 \\
0.70 & 620 & 49 \\
1.25 & 130 & 33 \\
\hline
\end{tabular}

Notes. Different flux values for the same source at the same wavelength from the same authors implies different apertures.

References. (33) Ibrahimov 1999; (37) Kenyon \& Hartmann 1991; (49) Mendoza V. 1971; (73) Simon et al. 1972.

(This table is available in its entirety in machine-readable and Virtual Observatory (VO) forms in the online journal. A portion is shown here for guidance regarding its form and content.)

This is particularly relevant to characterize the FU Orionis phenomenon.

Tables 3 and 4 list the parameters of the models that best fit the SEDs of the 24 analyzed objects. Table 3 corresponds to class II FUors, and Table 4 to class I FUors. In both cases we provide the envelope mass $\left(M_{\text {env }}\right)$ as an additional parameter. Although not independent, this parameter has been determined by other authors and thus provides a direct way to compare our results with others. In the last column we give sample values for class I and II parameters (Robitaille et al. 2007). Figures 1.1 to 1.25 show the best fit obtained in each case. Uncertainties in fluxes available in the literature are indicated with error bars, except when smaller than the size of the symbols.
Since FU Orionis stars are variable stars, when constructing their SEDs we attempted to use contemporary data whenever possible. This was particularly the case in the optical and NIR wavelengths, where the variations are larger. Five of the analyzed objects (V1515 Cyg, V1057 Cyg, L1551 IRS5, RNO 1B, and V1331 Cyg) show a significant dispersion in the observed fluxes, due to the variability of the source during the period of time covered by the data. For this reason, fluxes at different times were selected to construct individual SEDs. In the case of RNO 1B and V1331 Cyg, we defined two SEDs that were modeled independently. On the other hand, for V1515 Cyg, V1057 Cyg, and L1551 IRS5 the identification of fluxes corresponding to different observing periods was not useful to reduce their dispersion. Therefore, we chose to model the fluxes contemporary to the observed MIR spectra. For five of the analyzed sources (V1647 Ori, OO Ser, V2492 Cyg, HBC 722, and V2775 Ori) we can clearly distinguish two epochs, before and after the outburst. Consequently, two SEDs were modeled. In general, fluxes from the literature were obtained with different aperture sizes. For this reason, for the sources V1515 Cyg, FU Ori, V1057 Cyg, AR 6B, V346 Nor, and Re 50 N IRS, we show models corresponding to different apertures.

\section{ANALYSIS OF INDIVIDUAL SOURCES}

\subsection{Class II FU Orionis Stars}

\subsubsection{V1515 Cygni}

The outburst of V1515 Cygni, one of the three prototypes of the FUor class, was detected in the optical in 1950 and it has remained in an outburst stage since then (Herbig 1977). Goodrich (1987) suggested the presence of a molecular outflow associated with this object (see also Evans et al. 1994). Furthermore, they argued that the inclination angle of this source to the line of sight should be close to zero, according to the shape of the large-scale nebula associated with it. Weintraub et al. (1991) detected V1515 Cygni at 450, 800, and $850 \mu \mathrm{m}$, and the central source has an estimated luminosity between $77 L_{\odot}$ and $200 L_{\odot}$ (Sandell \& Weintraub 2001; Green et al. 2006). Kóspál (2011)

Table 3

Model Parameters for the Class II FU Orionis

\begin{tabular}{|c|c|c|c|c|c|c|c|c|c|c|}
\hline Parameter & $\begin{array}{c}\text { V1515 } \\
\text { Cyg }\end{array}$ & BBW 76 & $\begin{array}{c}\text { V1735 } \\
\text { Cyg }\end{array}$ & $\begin{array}{c}\text { V883 } \\
\text { Ori }\end{array}$ & $\mathrm{RNO} 1 \mathrm{~B}^{\mathrm{a}}$ & RNO 1C & PP 13S & $\begin{array}{c}\text { V1647 } \\
\text { Ori (pre) }\end{array}$ & $\begin{array}{c}\text { V1647 } \\
\text { Ori (post) }\end{array}$ & Class II \\
\hline$M_{*}\left(M_{\odot}\right) M_{*}\left(M_{\odot}\right)$ & 0.3 & 0.5 & 0.4 & 1.5 & 0.2 & 0.2 & 0.6 & 1.0 & 1.0 & 1.61 \\
\hline$R_{*}\left(R_{\odot}\right)$ & 2.0 & 3.0 & 3.0 & 2.5 & 1.8 & 1.8 & 2.5 & 1.5 & 1.5 & $\ldots$ \\
\hline$T_{*}(\mathrm{~K})$ & 5900 & 6500 & 5000 & 6000 & $6000(5600)$ & 6000 & 4800 & 4500 & 6000 & 4268 \\
\hline$\dot{M}\left(10^{-6} M_{\odot} \mathrm{yr}^{-1}\right)$ & 0.10 & 0.10 & 0.08 & 0.01 & 0.10 & 0.10 & 0.10 & 0.30 & 0.10 & 0.01 \\
\hline$R_{\mathrm{c}}(\mathrm{AU})$ & 32 & 160 & 200 & 200 & 80 & 81 & 200 & 300 & 300 & 239 \\
\hline$R_{\min }(\mathrm{AU})$ & 0.47 & 0.42 & 0.56 & 0.19 & 0.04 & 0.04 & 0.12 & 0.06 & 0.11 & 1.16 \\
\hline$R_{\max }(\mathrm{AU})$ & 8200 & 4700 & 8000 & 3800 & 1000 & 6000 & 5000 & 2840 & 2840 & $\ldots$ \\
\hline$M_{\mathrm{env}}\left(M_{\odot}\right)$ & 0.050 & 0.003 & 0.900 & 0.240 & 0.370 & 0.370 & 0.120 & 0.000 & 0.000 & $\ldots$ \\
\hline$M_{\text {disk }}\left(M_{\odot}\right)$ & 0.13 & 0.08 & 0.20 & 0.30 & $0.20(0.01)$ & 0.20 & 0.24 & 0.40 & 0.40 & 0.03 \\
\hline$\dot{M}_{\text {disk }}\left(10^{-5} M_{\odot} \mathrm{yr}^{-1}\right)$ & 1.0 & 1.0 & 1.4 & 1.0 & $0.8(0.3)$ & 0.8 & 8.0 & 0.01 & 0.5 & 0.2 \\
\hline$\rho_{\mathrm{amb}}\left(10^{-22}\right)$ & 1.0 & 23.0 & 10.0 & 1.0 & 500.0 & 500.0 & 130.0 & 2.2 & 2.2 & $\ldots$ \\
\hline$\rho_{\text {cav }}\left(10^{-20}\right)$ & 1.00 & 0.30 & 0.80 & 7.90 & 100.00 & 100.00 & 19.00 & 3.00 & 3.00 & $\ldots$ \\
\hline A & 2.050 & 2.005 & 2.005 & 2.213 & 2.100 & 2.103 & 2.250 & 2.200 & 2.100 & $\ldots$ \\
\hline B & 1.050 & 1.005 & 1.005 & 1.213 & 1.100 & 1.103 & 1.250 & 1.200 & 1.100 & $\ldots$ \\
\hline$\theta\left({ }^{\circ}\right)$ & 25 & 48 & 55 & 5 & 10 & 5 & 20 & 7 & 7 & $\ldots$ \\
\hline$i\left(^{\circ}\right)$ & 25 & 60 & 76 & 18 & 85 & 83 & 50 & 60 & 60 & $\ldots$ \\
\hline
\end{tabular}

Notes.

${ }^{a}$ We obtained two models, corresponding to different observing periods. Between the brackets are the values that best reproduce the most recent data when they differ from those from the older data model.

b Averages values from Robitaille et al. (2007). 
Table 4

Model Parameters for the Class I FU Orionis

\begin{tabular}{|c|c|c|c|c|c|}
\hline Parameters & FU Ori & V1057 Cyg & $\mathrm{ZCMa}$ & AR $6 \mathrm{~A}$ & Class I $^{\mathrm{a}}$ \\
\hline$M_{*}\left(M_{\odot}\right)$ & 0.70 & 0.5 & 0.80 & 0.80 & 0.93 \\
\hline$R_{*}\left(R_{\odot}\right)$ & 5.00 & 3.6 & 2.00 & 5.48 & $\ldots$ \\
\hline$T_{*}(\mathrm{~K})$ & 6030 & 6000 & 6500 & 4100 & 3073 \\
\hline$\dot{M}\left(10^{-6} M_{\odot} \mathrm{yr}^{-1}\right)$ & 1.0 & 0.50 & 10.0 & 30.0 & 9.73 \\
\hline$R_{c}(\mathrm{UA})$ & 70 & 60 & 65 & 80 & 397 \\
\hline$R_{\min }\left(R_{*}\right)$ & 0.47 & 1.00 & 0.09 & 0.19 & 5.9 \\
\hline$R_{\max }(\mathrm{AU})$ & 10000 & 5200 & 16170 & 7900 & $\ldots$ \\
\hline$M_{\mathrm{env}}\left(M_{\odot}\right)$ & 0.138 & 0.015 & 1.470 & 0.200 & $\ldots$ \\
\hline$M_{\text {disk }}\left(M_{\odot}\right)$ & 0.010 & 0.10 & 0.100 & 0.340 & 0.01 \\
\hline$\dot{M}_{\text {disk }}\left(10^{-6} M_{\odot} \mathrm{yr}^{-1}\right)$ & 1.00 & 14.0 & 20.00 & 4.30 & 0.6 \\
\hline$\rho_{\mathrm{amb}}\left(10^{-22}\right)$ & 1.0 & 10.0 & 15.9 & 3.5 & $\ldots$ \\
\hline$\rho_{\text {cav }}\left(10^{-20}\right)$ & 0.01 & 0.01 & 0.25 & 0.33 & $\cdots$ \\
\hline A & 2.090 & 2.005 & 2.064 & 2.200 & $\ldots$ \\
\hline B & 1.090 & 1.005 & 1.064 & 1.200 & $\ldots$ \\
\hline$\theta\left({ }^{\circ}\right)$ & 70 & 35 & 25 & 25 & $\ldots$ \\
\hline$i\left(^{\mathrm{o}}\right)$ & 75 & 30 & 32 & 73 & $\ldots$ \\
\hline Parameters & AR 6B & L1551 IRS 5 & V900 Mon & ISO-Cha I 192 & Class I $^{\mathrm{a}}$ \\
\hline$M_{*}\left(M_{\odot}\right)$ & 0.87 & 1.50 & 1.00 & 1.20 & 0.93 \\
\hline$R_{*}\left(R_{\odot}\right)$ & 5.50 & 2.50 & 1.50 & 6.10 & $\ldots$ \\
\hline$T_{*}(\mathrm{~K})$ & 4100 & 4800 & 6400 & 5000 & 3073 \\
\hline$\dot{M}\left(10^{-6} M_{\odot} \mathrm{yr}^{-1}\right)$ & 7.0 & 10.0 & 4.0 & 5.0 & 9.73 \\
\hline$R_{c}(\mathrm{UA})$ & 80 & 200 & 300 & 60 & 397 \\
\hline$R_{\min }\left(R_{*}\right)$ & 0.18 & 0.12 & 0.13 & 0.30 & 5.9 \\
\hline$R_{\max }(\mathrm{AU})$ & 7900 & 5000 & 2840 & 5000 & $\ldots$ \\
\hline$M_{\mathrm{env}}\left(M_{\odot}\right)$ & 0.010 & 0.170 & 0.027 & 0.180 & $\ldots$ \\
\hline$M_{\text {disk }}\left(M_{\odot}\right)$ & 0.370 & 0.200 & 0.200 & 0.150 & 0.01 \\
\hline$\dot{M}_{\text {disk }}\left(10^{-6} M_{\odot} \mathrm{yr}^{-1}\right)$ & 1.00 & 0.30 & 2.00 & 0.10 & 0.6 \\
\hline$\rho_{\mathrm{amb}}\left(10^{-22}\right)$ & 3.5 & 1.3 & 2.2 & 1.3 & $\ldots$ \\
\hline$\rho_{\text {cav }}\left(10^{-20}\right)$ & 0.33 & 1.90 & 30.00 & 1.90 & $\ldots$ \\
\hline A & 2.191 & 2.250 & 2.050 & 2.010 & $\ldots$ \\
\hline B & 1.191 & 1.250 & 1.050 & 1.010 & $\ldots$ \\
\hline$\theta\left({ }^{\circ}\right)$ & 15 & 33 & 50 & 20 & $\ldots$ \\
\hline$i\left(^{\circ}\right)$ & 72 & 70 & 30 & 50 & $\ldots$ \\
\hline Parameters & V346 Nor & OO Ser ${ }^{b}$ & $\operatorname{Re} 50$ N IRS 1 & V2492 Cyg $^{\text {b }}$ & Class I $^{\mathrm{a}}$ \\
\hline$M_{*}\left(M_{\odot}\right)$ & 0.30 & 0.70 & 1.00 & 1.20 & 0.93 \\
\hline$R_{*}\left(R_{\odot}\right)$ & 3.00 & $3.00(2.00)$ & 4.00 & $2.80 / 3.00(2.50)$ & $\ldots$ \\
\hline$T_{*}(\mathrm{~K})$ & 7000 & $6000(5000)$ & 6000 & $6100 / 6500(5000)$ & 3073 \\
\hline$\dot{M}\left(10^{-6} M_{\odot} \mathrm{yr}^{-1}\right)$ & 6.0 & 10.0 & 12.4 & 1.0 & 9.73 \\
\hline$R_{c}(\mathrm{UA})$ & 90 & 200 & 30 & 500 & 397 \\
\hline$R_{\min }\left(R_{*}\right)$ & 0.24 & 0.22 & 0.70 & 0.01 & 5.9 \\
\hline$R_{\max }(\mathrm{AU})$ & 5200 & 10000 & 6080 & 2000 & $\ldots$ \\
\hline$M_{\mathrm{env}}\left(M_{\odot}\right)$ & 0.300 & 0.910 & 0.280 & 0.200 & $\ldots$ \\
\hline$M_{\text {disk }}\left(M_{\odot}\right)$ & 0.050 & 0.010 & 0.060 & 0.030 & 0.01 \\
\hline$\dot{M}_{\text {disk }}\left(10^{-6} M_{\odot} \mathrm{yr}^{-1}\right)$ & 9.00 & $50.00(1.00)$ & 1.30 & $0.40(0.10)$ & 0.6 \\
\hline$\rho_{\mathrm{amb}}\left(10^{-22}\right)$ & 10.0 & 57.0 & 4.2 & 17.0 & $\ldots$ \\
\hline$\rho_{\text {cav }}\left(10^{-20}\right)$ & 1.00 & 17.00 & 1.50 & 17.00 & $\ldots$ \\
\hline A & 2.050 & 2.250 & 2.174 & 3.300 & $\ldots$ \\
\hline B & 1.050 & 1.250 & 1.174 & 1.500 & $\ldots$ \\
\hline$\theta\left({ }^{\mathrm{o}}\right)$ & 20 & 70 & 40 & 70 & $\ldots$ \\
\hline$i\left(^{\mathrm{o}}\right)$ & 5 & 5 & 15 & 13 & $\ldots$ \\
\hline Parameters & V1331 Cyg ${ }^{\mathrm{b}}$ & $\mathrm{HBC} 722^{\mathrm{b}}$ & Par 21 & V2775 Orib & Class I $^{\mathrm{a}}$ \\
\hline$M_{*}\left(M_{\odot}\right)$ & 0.8 & 1.00 & 1.00 & 0.50 & 0.93 \\
\hline$R_{*}\left(R_{\odot}\right)$ & 2.0 & $1.90(1.50)$ & 3.20 & $1.90(1.70)$ & $\ldots$ \\
\hline$T_{*}(\mathrm{~K})$ & $6600(5770)$ & $7100(5600)$ & 8700 & $6800(5600)$ & 3073 \\
\hline$\dot{M}\left(10^{-6} M_{\odot} \mathrm{yr}^{-1}\right)$ & 0.80 & 1.0 & 1.5 & $0.7(3.0)$ & 9.73 \\
\hline$R_{c}(\mathrm{UA})$ & 100 & 51 & 60 & 36 & 397 \\
\hline$R_{\min }\left(R_{*}\right)$ & 0.19 & 0.09 & 0.89 & 0.09 & 5.9 \\
\hline$R_{\max }(\mathrm{AU})$ & 8000 & 10000 & 5200 & 30200 & $\ldots$ \\
\hline$M_{\mathrm{env}}\left(M_{\odot}\right)$ & 0.120 & 0.060 & 0.020 & 0.300 & $\ldots$ \\
\hline$M_{\text {disk }}\left(M_{\odot}\right)$ & $0.10(0.02)$ & 0.100 & 0.300 & $0.150(0.008)$ & 0.01 \\
\hline$\dot{M}_{\text {disk }}\left(10^{-6} M_{\odot} \mathrm{yr}^{-1}\right)$ & $2.0(0.1)$ & $4.00(0.40)$ & 4.00 & $10.00(0.60)$ & 0.6 \\
\hline$\rho_{\mathrm{amb}}\left(10^{-22}\right)$ & 4.2 & 5.0 & 10.0 & 5.0 & $\ldots$ \\
\hline$\rho_{\text {cav }}\left(10^{-20}\right)$ & 1.50 & 0.01 & 1.00 & 0.01 & $\ldots$ \\
\hline
\end{tabular}


Table 4

(Continued)

\begin{tabular}{lccccc}
\hline \hline Parameters & V1331 Cyg & HBC 722 & Par 21 & ${\text { V2775 } \text { Ori }^{\mathrm{b}}}^{\text {Class I }^{\mathrm{a}}}$ \\
\hline $\mathrm{A}$ & 2.250 & 2.100 & 2.050 & 2.205 & $\ldots$ \\
$\mathrm{B}$ & 1.250 & 1.100 & 1.050 & 1.205 & $\ldots$ \\
$\theta\left({ }^{\circ}\right)$ & 50 & 30 & 80 & 35 & $\ldots$ \\
$i\left({ }^{\circ}\right)$ & 2 & 85 & 79 & 60 & $\ldots$ \\
\hline
\end{tabular}

Notes.

${ }^{a}$ Averages values from Robitaille et al. (2007).

${ }^{\mathrm{b}}$ For these objects we obtained two models associated with two different periods of observations. We indicate between brackets the best model parameters corresponding to the most recent data when they differ from those obtained form older observations (see Figures 1.19, $1.21,1.22,1.23$, and 1.25).

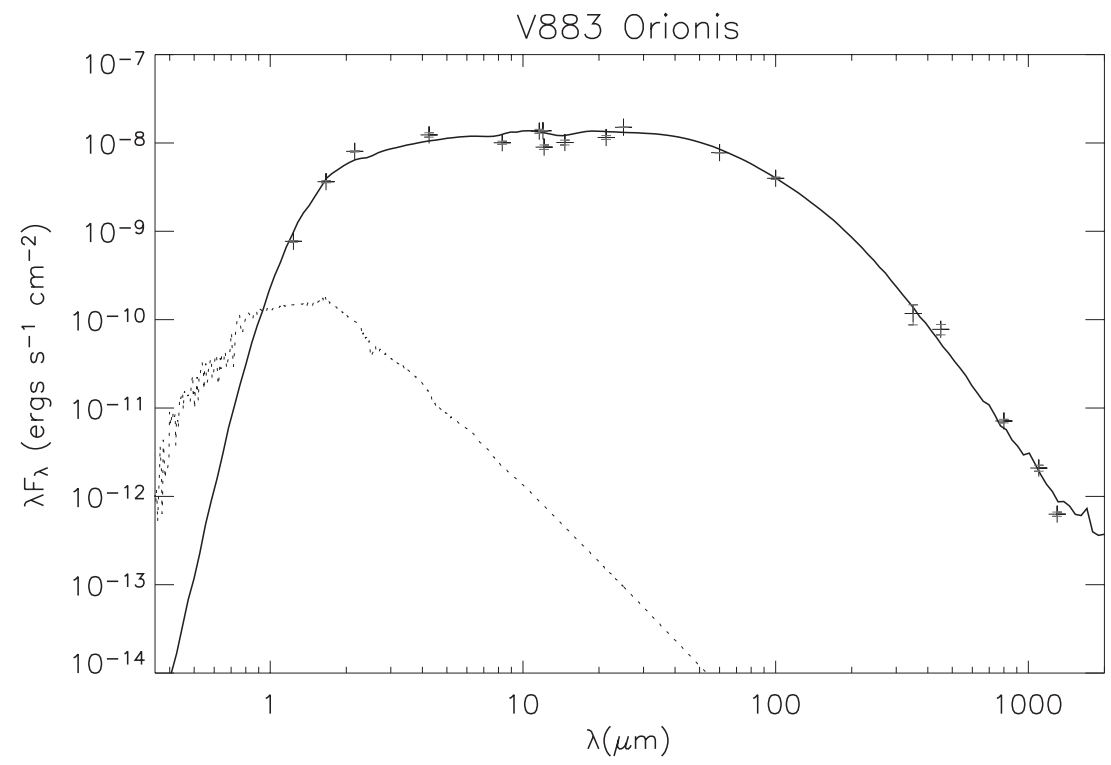

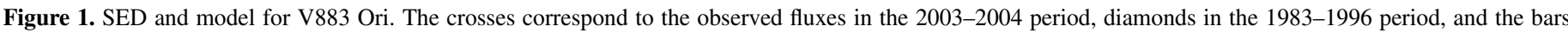

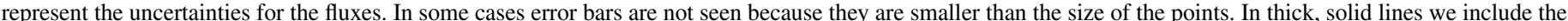

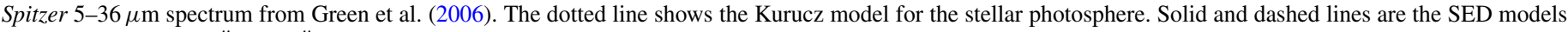
for aperture sizes of $60^{\prime \prime}$ and $11^{\prime \prime}$, respectively.

(The complete figure set (25 images) is available in the online journal.)

obtained ${ }^{13} \mathrm{CO}$ maps of V1515 Cyg that show an arc-shaped emission structure.

The SED of V1515 Cyg has two peaks, at $\sim 1.5 \mu \mathrm{m}$ and $\sim 60 \mu \mathrm{m}$. The Spitzer-IRS spectrum in the $5-36 \mu \mathrm{m}$ range shows the presence of silicate in emission at $\sim 9.7 \mu \mathrm{m}$ (Green et al. 2006). The observed fluxes from $4 \mu \mathrm{m}$ to $200 \mu \mathrm{m}$ show a relatively large dispersion. This is mainly caused by the difference in the time when observations were obtained, which can be seen in Figure 1.1, where the crosses correspond to the fluxes observed between 2003 and 2004, and diamonds correspond to the observations obtained between 1983 and 1996.

The best fit for V1515 Cyg (Table 3) corresponds to the fluxes obtained from 2003 to 2004 (crosses in Figure 1.1), which are contemporaneous to the Spitzer spectrum. The 1983-1996 data (diamonds in Figure 1.1) have a large dispersion, which makes the modeling more difficult. We adopted the published spectral type for this source (G2-G5; see Table 1 in Kolotilov \& Petrov 1983), which corresponds to a temperature in the 5860-5770 K range (Kenyon \& Hartmann 1995).

Figure 1.1 shows our best model for two different apertures, $60^{\prime \prime}$ (solid line) and 11" (dashed line). The latter aperture value is similar to that used in the extraction of the spectrum (Green et al. 2006).
Sandell \& Weintraub (2001) determined an upper limit for the disk mass of V1515 Cyg of $\sim 0.13 M_{\odot}$, from observations at $1.3 \mathrm{~mm}$. Lodato \& Bertin (2001) estimated an opening angle for the cavity $\theta \sim 20^{\circ}-28^{\circ}$, a disk mass accretion rate $\dot{M}_{\text {disk }}=1.0 \times 10^{-5} M_{\odot} \mathrm{yr}^{-1}$, and a disk mass $M_{\text {disk }}=$ 0.9-1.5 $M_{\odot}$. Green et al. (2006) modeled the NIR and MIR SED, as well as the Spitzer spectrum, and derived a maximum temperature for the central source of $7710 \mathrm{~K}$, and $M_{*} \dot{M}_{\text {disk }}=$ $3.5 \times 10^{-5} M_{\odot}^{2} \mathrm{yr}^{-1}$. Zhu et al. (2008) estimated an inclination angle $i=0^{\circ}$ and a central star mass $M_{*}=0.3 M_{\odot}$, a stellar radius $R_{*}=2.8 R_{\odot}$, a disk inner radius $R_{\min }=0.25 \mathrm{AU}$, and a value of $M_{*} \dot{M}_{\text {disk }}=1.3 \times 10^{-5} M_{\odot}^{2} \mathrm{yr}^{-1}$.

Table 3 lists our best fit parameters for V1515 Cyg. The stellar temperature is lower than the maximum determined by Green et al. (2006), but consistent with the spectral type. The disk mass $\left(0.13 M_{\odot}\right)$ is also lower than that obtained by Lodato $\&$ Bertin $\left(2001, M_{\text {disk }}=0.91-1.52 M_{\odot}\right)$. Furthermore, from our disk mass accretion rate $\dot{M}_{\text {disk }}=1.0 \times 10^{-5} M_{\odot} \mathrm{yr}^{-1}$ and stellar mass, $M_{*}=0.3 M_{\odot}$, we derived $M_{*} \dot{M}_{\text {disk }}=1.1 \times$ $10^{-5} M_{\odot}^{2} \mathrm{yr}^{-1}$. This value is lower than previous estimations by other authors, nevertheless the stellar mass agrees with the determination of Zhu et al. (2008) and the disk massaccretion rate matches the estimation of Lodato \& Bertin (2001). 
The values of $\theta=25^{\circ}$ and $R_{*}=2.0 R_{\odot}$ listed in Table 3 are in good agreement with those determined by Lodato \& Bertin (2001) and Zhu et al. (2008), respectively.

\subsection{2. $B B W 76$}

BBW 76, also known as BRAN 76 and IRAS 07486-3258 was identified by Reipurth (1985b) as a FUor. Later, Eisloeffel et al. (1990) confirmed this identification based on high-resolution optical spectra, in particular by the P-Cygni Balmer line profiles and absorption line widths, similar to FUors prototypes. In addition, Reipurth et al. (2002a) identified several other observational properties, such as the change of the spectral type toward later types with increasing wavelength, which is common to well known FU Ori stars. BBW 76 is not associated with any known molecular outflow (Sandell \& Weintraub 2001), and Green et al. (2006) suggested that BBW 76 might be a class I object.

Figure 1.2 shows the SED of BBW 76, which presents a maximum at around $1.5 \mu \mathrm{m}$. The 5-36 $\mu \mathrm{m}$ Spitzer-IRS spectrum shows a strong silicate absorption at $\sim 9.7 \mu \mathrm{m}$ (Green et al. 2006). The best model obtained for BBW 76 is shown in Figure 1.2 (solid line), and its parameters are in Table 3. This model successfully reproduces the observed fluxes, however, at $\sim 10 \mu \mathrm{m}$ the observed fluxes do not match the Spitzer spectrum and also show a moderate dispersion. In the SED modeling, more weight was given to spectrum than to the individual flux values.

Sandell \& Weintraub (2001) determined a disk mass of $0.15 M_{\odot}$ from sub-mm observations. Green et al. (2006) estimated a maximum temperature of $7710 \mathrm{~K}$, a disk inner radius $R_{\text {min }}=3.9 R_{\odot}$, a luminosity $L_{*}=1.8 L_{\odot}$, and $M_{*} \dot{M}_{\text {disk }}=$ $7.2 \times 10^{-5} M_{\odot}^{2} \mathrm{yr}^{-1}$. Zhu et al. (2008) obtained $R_{*}=4.6 R_{\odot}$, $R_{\text {min }}=0.64 \mathrm{AU}$, and $M_{*} \dot{M}_{\text {disk }}=8.1 \times 10^{-5} M_{\odot}^{2} \mathrm{yr}^{-1}$, with an inclination angle $i=50^{\circ}$.

The stellar radius $\left(3.0 R_{\odot}\right)$ and the disk inner radius $\left(R_{\min }=\right.$ $0.42 \mathrm{AU})$ we derive agree with the values obtained by $\mathrm{Zhu}$ et al. (2008), however our modeled disk mass $\left(0.08 M_{\odot}\right)$ is less than that determined by Sandell \& Weintraub (2001, $\left.M_{\text {disk }} \sim 0.15 M_{\odot}\right)$. The disk mass accretion rate is $\dot{M}_{\text {disk }}=$ $1 \times 10^{-5} M_{\odot} \mathrm{yr}^{-1}$, then $M_{*} \dot{M}_{\text {disk }}=8 \times 10^{-7} M_{\odot}^{2} \mathrm{yr}^{-1}$, is two orders of magnitude lower than that obtained by other authors.

\subsubsection{V1735 Cygni}

V1735 Cygni is located in the IC 5146 stellar cluster in L1031, at a distance of $900 \mathrm{pc}$ (Hilton \& Lahulla 1995), and it is also known as Elias 1-12. Elias (1978) identified V1735 Cygni as a FU Orionis type variable. Its outburst took place between $\sim 1957$ and 1965 (Hartmann \& Kenyon 1996). V1735 Cyg is associated with a high-mass molecular outflow (Levreault 1983), and has a luminosity of $25 L_{\odot}$.

The observed SED is shown in Figure 1.3 and reveals two peaks, one around $1 \mu \mathrm{m}$ and the other in the $60-100 \mu \mathrm{m}$ range. Although the observed fluxes cover the spectral range from the infrared to close to $100 \mu \mathrm{m}$, data at longer wavelengths are scarce, which results in an uncertain behavior of the SED in that spectral range. This complicates the determination of reliable parameters for the envelope. Nevertheless, the best model obtained (solid line, Figure 1.3) reproduces the observed SED satisfactorily well.

Table 3 shows the parameters corresponding to the model presented in Figure 1.3. From observations in the mm, Sandell $\&$ Weintraub (2001) derived a mass of $0.42 M_{\odot}$, that they associate with the disk. However, our model disk mass $M_{\text {disk }}=$ $0.20 M_{\odot}$ is roughly half of that value. The envelope mass we obtain is $M_{\mathrm{env}}=0.9 M_{\odot}$, which suggests that the envelope might contribute to the mass value determined by Sandell $\&$ Weintraub (2001). The disk mass accretion rate $\dot{M}_{\text {disk }}=$ $1.4 \times 10^{-5} M_{\odot} \mathrm{yr}^{-1}$ agrees with previous estimates for other class II FU Orionis.

\subsubsection{V883 Orionis}

Strom \& Strom (1993) reported V883 Orionis as a FU Orionis object in the IC 430 nebula of the Orionis region. It has a luminosity of $400 L_{\odot}$ and is not associated with any known molecular outflow (Sandell \& Weintraub 2001). Figure 1 (1.4 in the online journal) shows its observed SED. The dispersion of the fluxes is small and it is relatively well covered in the region beyond $100 \mu \mathrm{m}$. However, few fluxes are available in the NIR region. This SED is rather flat and without any distinguishable feature.

Table 3 lists the parameters corresponding to the model in Figure 1 (1.4 in the online journal). The disk mass $M_{\text {disk }}=$ $0.3 M_{\odot}$ is consistent with the $0.39 M_{\odot}$ estimated by Sandell \& Weintraub (2001) from millimeter (mm) observations. The disk mass accretion rate $\dot{M}_{\text {disk }}=1 \times 10^{-5} M_{\odot} \mathrm{yr}^{-1}$ matches the values derived for the other class II FU Orionis objects.

\subsection{5. $R N O 1 B$}

This object, also as known as V710 Cas, was identified as a FUor by Staude \& Neckel (1991). It is located in the L1287 dark cloud at a distance of 850 pc (Yang et al. 1991), and constitutes a binary system with RNO 1C (also a FUor, see following section), for which Quanz et al. (2006) estimated a separation of 5000 AU. According to Snell et al. (1990) and Yang et al. (1991), RNO 1B is associated with a highmass molecular outflow. However, McMuldroch et al. (1995) identified RNO $1 \mathrm{C}$ as the driving source of the outflow.

Figure 1.5 shows the observed SED of RNO 1B. The fluxes have a relatively large dispersion, which can be attributed to the different epochs of observation. For this reason, the observations are divided into two periods, until 1995 (crosses) and after 1996 (asterisks). The SED of this object includes the Spitzer-IRS 5-14 $\mu \mathrm{m}$ spectrum (Quanz et al. 2007b). The solid line in Figure 1.5 indicates the model for pre-1995 fluxes, and a dotted-dashed line indicates the post-1996 model.

In Table 3 we list the parameters corresponding to the data obtained before 1995, and in brackets we indicate the values obtained from the fluxes observed after 1996 whenever they differ. Comparing the results from the two epochs, we see that the disk mass, the temperature, and the disk mass accretion rate have all decreased. Particularly, the disk mass has decreased by a factor 20. The decrease in the disk mass accretion rate might be an indicator that the central star would be in its way to enter the T Tauri or class II evolutionary stage. However, this should be confirmed by more detailed determinations of $\dot{M}_{\text {disk }}$. It is worthwhile to mention that $\dot{M}_{\text {disk }} \sim 1.0 \times 10^{-5} M_{\odot} \mathrm{yr}^{-1}$ at the time of highest brightness, which is the same order of magnitude as for other class II FUor sources.

\subsection{6. $R N O 1 C$}

RNO 1C was identified as a FU Orionis type star by Kenyon et al. (1993b). As mentioned before, RNO 1C and RNO 1B form a binary system in which both stars are FU Orionis variables. Figure 1.6 shows the observed SED for RNO 1C and the 5-14 $\mu \mathrm{m}$ Spitzer-IRS spectrum obtained by Quanz et al. 
(2007b). There are no observed fluxes around $100 \mu \mathrm{m}$ available in the literature, thus the behavior of the SED in that spectral region is very uncertain. Furthermore, the model reproduces well the shape of the Spitzer spectrum, but fails to reproduce individual fluxes around these wavelengths.

The parameters derived from this model are shown in Table 3. The outer radius value $R_{\max }=6000 \mathrm{AU}$ is in agreement with the $\sim 5000$ AU determined by McMuldroch et al. (1995) for the size of the envelope using CS molecular line observations. The disk mass accretion rate $\dot{M}_{\text {disk }}=8.0 \times 10^{-6} M_{\odot} \mathrm{yr}^{-1}$ agrees with the expected value for these types of objects.

\subsection{7. $P P 13 S$}

PP 13S is a protostar (Tapia et al. 1997; Sandell \& Weintraub 2001; Tsukagoshi et al. 2005) embedded in the small, dark cloud L1473, at a distance of 350 pc (Cohen et al. 1983). This source is associated with a bipolar molecular outflow traced by the $\mathrm{CO}(2-1)$ and $\mathrm{CO}(1-0)$ transitions (Sandell \& Aspin 1998; Tsukagoshi et al. 2005). Sandell \& Aspin (1998) identified PP 13S as a FU Orionis object from the broad and deep shape of the $\mathrm{CO}$ absorption band at $2.3 \mu \mathrm{m}$.

The SED (see Figure 1.7) has a maximum around $8 \mu \mathrm{m}$. However, the lack of data between $10 \mu \mathrm{m}$ and $200 \mu \mathrm{m}$ makes its shape uncertain. Nevertheless, our best SED model (Figure 1.7, solid line) reproduces satisfactorily the observed fluxes.

From NIR images, sub-mm continuum, and CO line observations Sandell \& Aspin (1998) suggested the existence of a disk associated with PP $13 \mathrm{~S}$ with an inclination of $40^{\circ}$ with respect to the line of sight. Tsukagoshi et al. (2005) estimated for PP $13 \mathrm{~S}$ an envelope mass $M_{\text {env }} \sim 0.27 M_{\odot}$, a mass accretion rate $\dot{M} \sim 5 \times 10^{-6} M_{\odot} \mathrm{yr}^{-1}$, and an inclination $i \sim 59^{\circ}$, from the mm continuum data and $\mathrm{C}^{18} \mathrm{O}(1-0)$ observations.

Table 3 presents the parameters derived from the model of the SED shown in Figure 1.7. The inclination angle $i=50^{\circ}$ is in agreement with previous determinations. The disk mass accretion rate value of $8 \times 10^{-6} M_{\odot} \mathrm{yr}^{-1}$, as well as the envelope mass $M_{\text {env }}=0.12 M_{\odot}$, agree with the values determined by Tsukagoshi et al. (2005).

\subsubsection{V1647 Orionis}

This source is located in the Lynds 1630 dark cloud in M78, at a distance of $400 \mathrm{pc}$, and illuminates the McNeil's reflection nebula (Lis et al. 1999). Two outbursts have been registered for this source. The first occurred between 2003 October and 2004 February, for which pre- and post-outburst observations are available (Briceño et al. 2004; Ábrahám et al. 2004a; Reipurth \& Aspin 2004b; McGehee et al. 2004; Andrews et al. 2004; Walter et al. 2004). The second outburst took place from 2008 to 2009 (Itagaki et al. 2008; Kun 2008).

Aspin et al. (2008) obtained optical, NIR, and MIR observations for V1647 Ori after the first outburst. Based on the relative long outburst and the detection of the $\mathrm{CO}$ overtone in absorption, these authors suggested its classification as a FUor. Later, Aspin et al. (2009) observed a very weak CO overtone bandhead absorption when the star was exhibiting a second brightness increase in 2008 August.

Aspin (2011a) observed this source in the NIR and noticed that the star remained in an outburst state during the 2008-2011 period, supporting the hypothesis of relatively long outbursts and thus the FUor classification. However, Aspin et al. (2006) suggested that V1647 Ori may be an EXOR variable. In particular a NIR spectrum, taken after the second outburst, shows the $\mathrm{CO}$ overtone bandheads in emission in addition to other emission lines in the optical and NIR (Aspin et al. 2010; Aspin 2011a), which are features commonly found in EXOR variables. In summary, V1647 Ori shows photometrical properties similar to FUors, and spectroscopic characteristics common to EXOR variables (Aspin 2011a; Semkov \& Peneva 2012).

In Figures 1.8 and 1.9 we show the SED before the 2003-2004 outburst and the SED for the post-outburst period between 2004 and 2008. For the period after the second outburst in 2008 there are currently not enough data to construct an SED. Table 3 lists the parameter values for both modeled SEDs, pre- and post-first outburst.

Reipurth \& Aspin (2004a) obtained an inclination angle $i=$ $30^{\circ}$ and an opening angle $\theta=60^{\circ}$, from the analysis of Gemini images. Muzerolle et al. (2005) modeled the SEDs before and after the 2003-2004 outburst, adopting a flat accretion disk (i.e., without flaring), a stellar mass $M_{*}=0.5 M_{\odot}$, and a stellar radius $R_{*}=2.0 R_{\odot}$. They obtained a mass accretion rate $\dot{M} \sim 10^{-6} M_{\odot} \mathrm{yr}^{-1}$ and an envelope total mass of $M_{\text {env }}=3 \times 10^{-3} M_{\odot}$ for the SED before the outburst. From the post-outburst SED, on the other hand, they derived a disk mass accretion rate $\dot{M}_{\text {disk }} \sim 10^{-5} M_{\odot} \mathrm{yr}^{-1}$, assuming that the bolometric luminosity is dominated by the accretion luminosity. Pre- and post-first-outburst mass accretion rates derived by Acosta-Pulido et al. (2007) are $\dot{M}=5 \times 10^{-7} M_{\odot} \mathrm{yr}^{-1}$ and $\dot{M}=1-7 \times 10^{-6} M_{\odot} \mathrm{yr}^{-1}$, respectively, and an inclination angle $i \sim 61^{\circ}$.

Aspin et al. (2008) used optical, NIR, and MIR observations after the outburst and estimated a $T_{\text {eff }} \sim 3800 \mathrm{~K}$ and a $R_{*} \sim 5 R_{\odot}$, together with a stellar mass value $M_{*} \sim 0.8 M_{\odot}$, this time from the position of V1647 Ori in the HR diagram. They also estimated a value $\dot{M}_{\text {disk }}=1.0 \pm 0.5 \times 10^{-6} M_{\odot} \mathrm{yr}^{-1}$ for the disk mass accretion rate, and $\theta=65^{\circ}, i=30^{\circ}$ for the cavity opening angle and the inclination angle to the line of sight, respectively.

For the second outburst period (i.e., 2008-2009), Aspin (2011a) derived a disk mass accretion rate $\dot{M}_{\text {disk }}=4 \pm 2 \times$ $10^{-6} M_{\odot} \mathrm{yr}^{-1}$, similar to that obtained by Aspin et al. (2008) for the first outburst.

When comparing our results for the pre- and after-outburst SEDs, we see that the stellar temperature and the mass accretion rates of the disk and the envelope all increased during the outburst (see Table 3 ). In particular, $\dot{M}_{\text {disk }}$ increased by an order of magnitude from $0.1 \times 10^{-6} M_{\odot} \mathrm{yr}^{-1}$ to $5 \times 10^{-6} M_{\odot} \mathrm{yr}^{-1}$. On the other hand, the stellar mass and the envelope mass, as well as the geometrical parameters $i=60^{\circ}$ and $\theta=7^{\circ}$, remain unchanged.

Our results derived from the SEDs analysis before and after the 2003-2004 outburst are, in general, in agreement with those obtained by Muzerolle et al. (2005) and Acosta-Pulido et al. (2007, see Figures 1.8 and 1.9, and Table 3). In particular, our values for the inclination angle and the disk mass accretion range for both pre- and post-outburst models agree very well with those derived by Acosta-Pulido et al. (2007). Furthermore, our determination for the disk mass accretion rate is also comparable to the value obtained by Muzerolle et al. (2005) for this source after the first outburst.

The stellar mass derived from our analysis is similar to the value obtained by Aspin et al. (2008), and our value of $5.0 \times 10^{-6} M_{\odot} \mathrm{yr}^{-1}$ for the disk mass accretion rate after the outburst is on the same order as the $1.0 \pm 0.5 \times 10^{-6} M_{\odot} \mathrm{yr}^{-1}$ they derived. However, the inclination angle $\left(i=60^{\circ}\right.$ versus $\left.30^{\circ}\right)$, as well as the opening angle $\left(\theta=7^{\circ}\right.$ versus $\left.65^{\circ}\right)$, differ. 
Moreover, Aspin (2011b) derived a disk mass accretion for the second outburst that is similar with our determination.

Our model parameters derived from the SED after the outburst and the results obtained by other authors agree with those expected for a class II FUors, with the exception of the disk mass accretion rate that turned out smaller than expected $\left(\dot{M}_{\text {disk }}=2 \times 10^{-5} M_{\odot} \mathrm{yr}^{-1}\right.$ versus $10^{-4} M_{\odot} \mathrm{yr}^{-1} ;$ Hartmann \& Kenyon 1996). However, as pointed out by Aspin (2011a) this star shows several observational properties common to EXOR variables.

\subsection{Class I FU Orionis Stars}

\subsubsection{FU Orionis}

This source is one of the three prototypes of the class. The outburst was observed in 1937 with a luminosity of $340 L_{\odot}$ (Sandell \& Weintraub 2001). FU Ori is a binary system with a separation of 217 AU (Malbet et al. 2005; Quanz et al. 2006), and has no associated optical jet or molecular outflow (Evans et al. 1994).

Its SEDs, shown in Figure 1.10, present two peaks: one at $\sim 1.5 \mu \mathrm{m}$ and the other at $\sim 100 \mu \mathrm{m}$. The Spitzer-IRS $(5-36 \mu \mathrm{m})$ spectrum displays a silicate emission between $10 \mu \mathrm{m}$ and $18 \mu \mathrm{m}$ (Green et al. 2006). Whereas the Spitzer spectrum gives a good constraint for the MIR region of the SED, fluxes at sub-mm and $\mathrm{mm}$ wavelengths are scarce and show a high dispersion.

Figure 1.10 shows the best model obtained for FU Ori. For this source we adopted $T_{*} \sim 6030 \mathrm{~K}$, in agreement with its G0 spectral type (see Table 1 in Kenyon et al. 2000), and the calibration of (Kenyon \& Hartmann 1995). We plot the models corresponding to two apertures: 60" (continuous line) and 20" (dashed line). The latter aperture is similar to that used by Green et al. (2006) to extract the spectrum.

Several authors have analyzed this object. Kenyon et al. (1988) adopted a stationary accretion disk model and reproduced both the SED and the observed line profiles. They derived a stellar mass of $0.37 M_{\odot}$ and a temperature of $7200 \mathrm{~K}$. In addition, they estimated $M_{*} \dot{M}_{\text {disk }}=0.5-4.0 \times 10^{-4} M_{\odot}^{2} \mathrm{yr}^{-1}$ for $\cos i=0.5$. Popham et al. (1996) fixed the value of the stellar mass at $0.7 M_{\odot}$ and used an accretion disk with a boundary layer to model both optical spectra and line profiles. For $\cos i=0.5$ they derived $M_{*} \dot{M}_{\text {disk }}=1.4 \times 10^{-4} M_{\odot}^{2} \mathrm{yr}^{-1}$.

More recently, Sandell \& Weintraub (2001) used observations in the sub-mm to estimate an upper limit for the disk mass of $0.02 M_{\odot}$. Lodato \& Bertin (2001), in turn, modeled the SED of FU Orionis using a self-gravitant accretion disk and obtained $M_{*} \dot{M}_{\text {disk }}=5.2 \times 10^{-5} M_{\odot}^{2} \mathrm{yr}^{-1}$ for $M_{*}=1 M_{\odot}$, $\cos i=0.65$, and $R_{\min }=8 R_{\odot}$. Subsequently, Lodato \& Bertin (2003) modeled the line profiles in addition to the SED, and derived $M_{*} \dot{M}_{\text {disk }}=10^{-4} M_{\odot}^{2} \mathrm{yr}^{-1}$, for $M_{*}=0.7 M_{\odot}$ and $\cos i=0.5$.

Malbet et al. (2005) used interferometric data in the NIR and determined $\dot{M}_{\text {disk }}=6.5 \times 10^{-5} M_{\odot} \mathrm{yr}^{-1}$. Green et al. (2006) modeled the SED and the Spitzer infrared spectrum with an accretion disk. They obtained $M_{*} \dot{M}_{\text {disk }}=1.0 \times 10^{-4} M_{\odot}^{2} \mathrm{yr}^{-1}$, for $R_{i}=0.58 \mathrm{AU}$ and $R_{c}=70 \mathrm{AU}$, adopting $M_{*}=0.3 M_{\odot}$ and a maximum stellar temperature of $7710 \mathrm{~K}$. Zhu et al. (2008) modeled the Spitzer-IRS spectrum of FU Ori and derived $i=55^{\circ}, R_{*}=5 R_{\odot}$, and $M_{*} \dot{M}_{\text {disk }}=7.4 \times 10^{-5} M_{\odot}^{2} \mathrm{yr}^{-1}$.

Our model of the SED of FU Orionis provides the parameters listed in Table 4. The central stellar mass $M_{*}=0.7 M_{\odot}$ agrees with previous determinations, especially with the value determined by Lodato \& Bertin (2003). The disk mass accretion rate we obtain is $\dot{M}_{\text {disk }}=10^{-5} M_{\odot} \mathrm{yr}^{-1}$ for a stellar mass $M_{*}=0.7 M_{\odot}$, therefore, $M_{*} \dot{M}_{\text {disk }}=0.7 \times 10^{-5} M_{\odot}^{2} \mathrm{yr}^{-1}$. This value is similar to those determined by Kenyon et al. (1988), Lodato \& Bertin (2003), and Zhu et al. (2008). However, it is lower than Lodato \& Bertin (2001). The disk mass is $0.01 M_{\odot}$, in agreement with the estimation of Sandell \& Weintraub (2001). Other parameters, such as $R_{*}\left(5.0 R_{\odot}\right), R_{\min }(0.47 \mathrm{AU})$, and $R_{c}$ (70 AU), are consistent with previous determinations by Green et al. (2006) and Zhu et al. (2008).

\subsubsection{V1057 Cygni}

Welin (1971) noticed that V1057 Cygni increased $~ 6$ mag in brightness in less than a year (1969-1971), which was reflected in a change of the spectral type of this object from M to early-A (Herbig 1977). Then, it gradually declined, fading by about 6 magnitudes in the following six years from the outburst. V1057 Cyg has an estimated luminosity between $170 L_{\odot}$ and $370 L_{\odot}$ (Sandell \& Weintraub 2001; Green et al. 2006), and it is associated with a molecular outflow (Evans et al. 1994) and surrounded by an envelope (Kóspál 2011).

Figure 1.11 shows our best model for V1057 Cygni. Because the data display a large scatter, in a first approximation we divided the observations in four epochs. We chose to model the fluxes contemporaneous to the Spitzer spectrum (diamonds in Figure 1.11) because older flux values show significant differences with the spectrum and do not provide a complete coverage in wavelength to well constrain our modeling attempt. The model for V1057 Cyg in Figure 1.11 is plotted for two apertures: 60" (solid line) and 11" (dotted-dashed line). The latter aperture is similar to that of the (Green et al. 2006) spectrum. In the region around $10 \mu \mathrm{m}$ the fluxes show dispersion independently of the observing epoch and the aperture used. For this reason, in our modeling we give more value to the spectrum than to the individual flux values. In addition, we adopted a spectral type corresponding to the time when the observations were obtained (F7/G3 I/II, Herbig et al. 2003), and derived a temperature $T \sim 5900-6500 \mathrm{~K}$, in concordance with Kenyon \& Hartmann (1995).

Kenyon et al. (1988) derived a maximum value for the inclination angle of $i=30^{\circ}$, a lower limit for the stellar mass of $M_{*}>0.1 M_{\odot}$, a radius $R_{*} \sim 4 R_{\odot}$ and $M_{*} \dot{M}_{\text {disk }} \sim 0.5-3 \times$ $10^{-4} M_{\odot}^{2} \mathrm{yr}^{-1}$. Popham et al. (1996) modeled both the SED and the line profiles and estimated $\dot{M}_{\text {disk }}=1.0 \times 10^{-4} M_{\odot} \mathrm{yr}^{-1}$ for $M_{*}=0.5 M_{\odot}$ and $R_{*}=5.03 R_{\odot}$, with an inclination angle of $30^{\circ}$. Lachaume (2004) modeled the SED of V1057 Cyg and obtained $M_{*} \dot{M}_{\text {disk }}=2 \times 10^{-5} M_{\odot}^{2} \mathrm{yr}^{-1}$, and $R_{\min }=2 R_{\odot}$. Finally, Green et al. (2006) adopted an inclination of $i=0^{\circ}$, and determined a maximum temperature $T_{*}<6590 \mathrm{~K}$, a $R_{\text {min }}=3.7 R_{\odot}$, and $M_{*} \dot{M}_{\text {disk }}=4.5 \times 10^{-5} M_{\odot}^{2} \mathrm{yr}^{-1}$.

Table 4 lists the best SED model parameters for V1057 Cygni. The derived stellar temperature is less than the maximum estimated by Green et al. (2006), whereas the stellar mass $\left(0.5 M_{\odot}\right)$ is in agreement with Kenyon et al. (1988) and Popham et al. (1996). Our disk mass accretion rate is $\dot{M}_{\text {disk }}=1.4 \times$ $10^{-4} M_{\odot} \mathrm{yr}^{-1}$, thus we derive $M_{*} \dot{M}_{\text {disk }}=7.0 \times 10^{-5} M_{\odot}^{2} \mathrm{yr}^{-1}$, in agreement with previous determinations.

\subsubsection{Z CMa}

This object is a close binary with a separation of $0^{\prime \prime} 1$ (Koresko et al. 1991; Thiebaut et al. 1995; Leinert et al. 1997), consisting of the two young stars Z CMa NW and Z CMa SE.

$\mathrm{Z}$ CMa NW is a Herbig Be star surrounded by a dusty cocoon with a hole (Szeifert et al. 2010; Canovas et al. 2012). 
This component has a mass of $12 M_{\odot}$ and a B8 spectral type (van den Ancker et al. 2004; Alonso-Albi et al. 2009). Z CMa SE has been classified as a FU Orionis object by Hartmann et al. (1989), based on the detection of a blueshifted $2 \mu \mathrm{m}$ CO firstovertone $v^{\prime}-v^{\prime \prime}=2-0$ and double-peaked optical absorption lines, with a velocity difference of about $100 \mathrm{kms}^{-1}$. This source has a luminosity of $420 L_{\odot}$ (Sandell \& Weintraub 2001), a stellar mass of $1.1 M_{\odot}$ (Pfalzner 2008), and a F5 spectral type (Kenyon et al. 1989). Canovas et al. (2012), using optical polarimetric images, found that the Z CMa system is surrounded by a common circumbinary envelope.

Z CMa was associated with a CO bipolar molecular outflow by Evans et al. (1994). More recently, Whelan et al. (2010) obtained adaptive-optics-assisted [Fe II] spectro-images that show the presence of two jets. In addition, observations carried out with OSIRIS at Keck revealed a parsec-scale wiggling outflow emanating from the Herbig Be star (Z CMa NW), suggesting that the central source may be double. Z CMa SE is, on the other hand, associated with a micro or small-scale jet (see also Canovas et al. 2012).

$\mathrm{Z}$ CMa has shown outburst events of less than one visual magnitude in a 5-10 yr timescale in 1987, 2000, and 2004 (van den Ancker et al. 2004; Grankin \& Artemenko 2009), typical of EXORS variables. In 2008 January, the brightness of Z CMa increased by about two visual magnitudes (Grankin \& Artemenko 2009). Based on spectropolarimetric observations, Szeifert et al. (2010) concluded that the outburst was associated with the Herbig Be component (Z CMa NW), which is embedded in a dusty cocoon. Moreover, the dynamical timescale of the wiggling outflow emanating from Z CMa NW (4-8 yr) agrees with the timescale between the outburst (Whelan et al. 2010).

Figure 1.12 shows the SED of Z CMa. The observed fluxes cover reasonably well the whole the spectral range, and the scatter in the observed fluxes is low. The best fit obtained (solid line) reproduces the shape of the observed SED satisfactorily well, with the exception of the NIR region. This is likely due to the binarity, since being a multiple system, the NIR portion of the SED has contributions from more than one of the stellar photospheres. For the modeling of this source we used a temperature value appropriated to the associated spectral type ( $T \sim 6440 \mathrm{~K}$, Kenyon \& Hartmann 1995).

Table 4 shows the best model parameters obtained for Z CMa. The stellar mass has a value $M_{*}=0.8 M_{\odot}$, similar to that determined by Pfalzner (2008). The disk mass accretion rate $\dot{M}_{\text {disk }}=2 \times 10^{-5} M_{\odot} \mathrm{yr}^{-1}$ is on the same order as that derived by these authors $\left(\dot{M}_{\text {disk }}=7.9 \times 10^{-5} M_{\odot} \mathrm{yr}^{-1}\right)$.

\subsection{4. $A R 6 A / 6 B$}

These stars are FU Orionis variables that lie in the NGC 2264 star-forming region, at a distance of $800 \mathrm{pc}$. They form a binary system with a separation of $\sim 2200$ AU (2". 8, Aspin \& Reipurth 2003). The source AR 6A has, in addition, a third companion AR 6C discovered by Aspin \& Reipurth (2003), with a separation of $700 \mathrm{AU}(0.85)$. Moriarty-Schieven et al. (2008) detected a molecular flow associated with AR 6A/6B.

Figures 1.13 and 1.14 show the SEDs of AR 6A and AR 6B, respectively. Fluxes compiled from the literature cover just the $1 \mu \mathrm{m}$ to $20 \mu \mathrm{m}$ range. The fluxes associated with AR 6B exhibit a large dispersion (Figure 1.14), because they were obtained with two different apertures. The best models for each of these sources reproduce the observed SEDs well. For AR 6B, the model is plotted for two apertures, 60" (solid line), and 30" (dotted-dashed line).
Table 4 lists the parameter values derived for the SEDs of $\mathrm{AR} 6 \mathrm{~A}$ and $\mathrm{AR} 6 \mathrm{~B}$. For the masses and temperatures of the central sources, we derived $M_{*}=0.80 M_{\odot}$ and $M_{*}=0.87 M_{\odot}$ for AR 6A and AR 6B, respectively, for sources both we obtain $T_{*} \sim 4100 \mathrm{~K}$. Parameters associated with the inner disk should be reasonably well constrained in the $1-20 \mu \mathrm{m}$ wavelength region. On the contrary, the external disk and the envelope parameters are poorly constrained in the models in Table 4 and Figures 1.13 and 1.14, since as mentioned before no fluxes for wavelength $>20 \mu \mathrm{m}$ are available for these sources.

\subsubsection{L1551 IRS5}

This object, also known as IRAS $04287+1801$, is a young protostellar binary system with a separation of 45 AU (Rodríguez et al. 1998), associated with a bipolar outflow seen in the optical and NIR (Snell et al. 1980; Mundt \& Fried 1983; MoriartySchieven \& Snell 1988; Stocke et al. 1988; Davis et al. 1995). L1551 IRS5 shows an optical spectrum characteristic of FU Orionis objects (Looney et al. 1997), for which it has been suggested that L1551 IRS5 belongs to this class. Sandell \& Weintraub (2001) estimated a mass of $0.23 M_{\odot}$ for the disk from observations in the $\mathrm{mm}$.

Figure 1.15 displays the observed SED of L1551 IRS5. Fluxes obtained from the literature cover well the spectral range between $1 \mu \mathrm{m}$ and $1200 \mu \mathrm{m}$, but have a modest dispersion around $100 \mu \mathrm{m}$. In the observed SED we include the Spitzer spectrum published by Quanz et al. (2007b), which shows a silicate absorption at $9.7 \mu \mathrm{m}$ typical of class I objects. It also shows $\mathrm{CO}_{2}$ in absorption at $6.85 \mu \mathrm{m}$. The best fit we obtained reproduces satisfactorily well the shape of the SED in the infrared region, as well as the spectrum around $10 \mu \mathrm{m}$. However, for wavelengths beyond $100 \mu \mathrm{m}$ the fit is relatively poor.

From the modeling of low resolution NIR images, Whitney et al. (1997) obtained a mass accretion rate $\dot{M}=$ $5 \times 10^{-6} M_{\odot} \mathrm{yr}^{-1}, R_{c}=30 \mathrm{AU}, \theta=20^{\circ}$, and $i \sim 70^{\circ}-90^{\circ}$. Osorio et al. (2003) determined $\dot{M}=7 \times 10^{-5} M_{\odot} \mathrm{yr}^{-1}$, and $R_{c}=150 \mathrm{AU}$ from the SED model of this source, whereas Robitaille et al. (2007) estimated an envelope mass accretion rate in the range between $5.5 \times 10^{-6} M_{\odot} \mathrm{yr}^{-1}$ and $3.0 \times 10^{-4} M_{\odot} \mathrm{yr}^{-1}$. At the same time, Gramajo et al. (2007) analyzed images in the $K$ - and $L$-bands, and obtained an inclination angle of $i=72^{\circ}-77^{\circ}$, an envelope mass accretion rate $\dot{M} \sim 5 \times 10^{-6} M_{\odot} \mathrm{yr}^{-1}$, a centrifugal radius $R_{c}=40-100 \mathrm{AU}$, and an opening angle $\theta=20^{\circ}$.

Table 4 lists the parameters for the best fit for L1551 IRS5 (see Figure 1.15). In general, these parameters are consistent with those determined by other authors. In particular, the inclination angle of $70^{\circ}$ agrees with that derived by Gramajo et al. (2007) and Whitney et al. (1997). However, the centrifugal radius is somewhat larger $\left(R_{c}=200 \mathrm{AU}\right.$ versus $\left.40-100 \mathrm{AU}\right)$, while the envelope mass accretion rate is an order of magnitude higher than that estimated by Gramajo et al. $\left(2007, \dot{M}=10^{-5} M_{\odot} \mathrm{yr}^{-1}\right.$ versus $\left.5 \times 10^{-6} M_{\odot} \mathrm{yr}^{-1}\right)$. Furthermore, the envelope mass accretion rate is less than that derived by Whitney et al. (1997), but it is consistent with the range of values estimated by Robitaille et al. (2007). On the other hand, the disk mass obtained from our SED modeling $\left(M_{\text {disk }}=0.2 M_{\odot}\right)$, is in good agreement with that obtained by Sandell \& Weintraub (2001).

$$
\text { 3.2.6. V900 Mon }
$$

V900 Mon, also known as 2MASS 06572222-0823176, was initially recognized as an eruptive variable (Thommes et al. 2011; Reipurth et al. 2012) in the L1656 small cloud located 
in a filamentary bridge between the Mon R2 complex and the CMa OB1 clouds, at a distance of $\sim 1100$ pc (Gregorio-Hetem 2008; Lombardi et al. 2011). This object is deeply embedded in a large cool envelope, with an estimated extinction of $A_{V} 13 \mathrm{mag}$ (Reipurth et al. 2012).

The same authors note that the spectra of V900 Mon has a striking resemblance to those of the prototype of the class, FU Ori, and by extension to the whole FUor class. The NIR spectra of V900 Mon shows a prominent CO bandhead absorption as well as large $\mathrm{H}_{2} \mathrm{O}$ broadband absorptions, suggesting a very late spectral type. In the optical, on the other hand, it shows characteristics suggesting an earlier than mid- to late-K spectral type. The appearance of the classic P-Cygny profile in lines such as $\mathrm{H} \alpha$ and the $\lambda 8662 \mathrm{Ca}$ II, as well as the $\lambda 6497 \mathrm{Ba}$ II feature, further support the inclusion of V900 Mon into the FUor class (Reipurth et al. 2012).

The photometric history of this object suggests that V900 Mon started its brightening sometime before the 1970s and is still ongoing. Reipurth et al. (2012) note that the brightness increase of this source is more consistent with that of the class-prototype V1515 Cyg. From Spitzer photometry, Reipurth et al. (2012) suggest that V900 Mon is a Class I source bordering the Class II sources, and the outburst appears to have occurred at an earlier evolutionary stage when the star was still partly embedded.

In Figure 1.16 we show the SED of V900 Mon covering the 1-200 $\mu \mathrm{m}$ spectral range. There are no sub-mm flux measurements available. The best models obtained reproduce the observed SEDs satisfactorily. The parameters for the model in Figure 1.16 are listed in Table 4 . We note that the lack of sub$\mathrm{mm}$ fluxes means that the envelope parameters of the model are not well constrained.

From our modeling, V900 Mon appears as a Class I source with an envelope mass accretion rate $M \sim 4.0 \times 10^{-6} M_{\odot} \mathrm{yr}^{-1}$. The values we obtain for the disk mass $\left(0.1 M_{\odot}\right)$ and disk mass accretion rate $\left(2.0 \times 10^{-6} M_{\odot} \mathrm{yr}^{-1}\right)$ are comparable with those derived for other Class I sources. Furthermore, the results we obtain are similar to those of V1647 Ori after its outburst (V1647 Ori (post) in Table 3), in agreement with the predictions of Reipurth et al. (2012).

\subsubsection{ISO-ChaI 192}

This class I protostar in the Chamaeleon I dark cloud is also known as GM Cha, [CCE98]2-41, ${ }^{7}$ DENIS-P J1109.5-7633, [PMK99] ISOCAM ChaI-Na2, ${ }^{8}$ and [PMK99] IR ChaI-Na1 (Cambresy et al. 1998; Persi et al. 1999; Gómez \& Mardones 2003), and it is associated with a CO molecular outflow (Mattila et al. 1989; Persi et al. 2007).

The SED of ISO-ChaI 192 is shown in Figure 1.17. For wavelengths greater than $30 \mu \mathrm{m}$ only a single flux value is available, at $70 \mu \mathrm{m}$. This affects the reliability of the parameters associated with the disk and the envelope.

Persi et al. (2007) modeled the SED of ISO-ChaI 192 using the code of Whitney et al. (2003a), and obtained an envelope mass accretion rate of $\dot{M}=1-3 \times 10^{-6} M_{\odot} \mathrm{yr}^{-1}$, a disk mass accretion rate of $\dot{M}_{\text {disk }}=1-7 \times 10^{-7} M_{\odot} \mathrm{yr}^{-1}$, a centrifugal radius $R_{c}=5-20 \mathrm{AU}$, an opening angle $\theta=5^{\circ}-30^{\circ}$, and an inclination angle $i=35^{\circ}-45^{\circ}$. These authors adopted fixed values for the stellar parameters $\left(M_{*}=0.55 M_{\odot}, R_{*}=2.5 R_{\odot}\right.$,

\footnotetext{
7 [CCE98] from Cambresy et al. (1998).

8 [PMK99] from Persi et al. (1999).
}

and $\left.T_{*}=3600 \mathrm{~K}\right)$, for the disk mass $\left(M_{\text {disk }}=0.15 M_{\odot}\right)$, and the disk inner radius $\left(R_{\min }=5.5 R_{*}\right)$.

The parameters for the SED model in Figure 1.17 are listed in Table 4 . The values derived for the inclination angle $\left(i=50^{\circ}\right)$ and the opening angle $\left(\theta=20^{\circ}\right)$ are consistent with those obtained by Persi et al. (2007). In addition, other parameters such as the disk mass accretion rate $\dot{M}_{\text {disk }}=1 \times 10^{-7} M_{\odot} \mathrm{yr}^{-1}$ and the envelope mass accretion rate $\dot{M}=5 \times 10^{-6} M_{\odot} \mathrm{yr}^{-1}$ agree with those derived by these authors. Nevertheless, the stellar parameters we obtain for the central source correspond to a more massive $\operatorname{star}\left(M_{*}=1.2 M_{\odot}\right.$ versus $0.55 M_{\odot}, R_{*}=6.1 R_{\odot}$ versus $2.5 R_{\odot}$, and $T_{*}=5000 \mathrm{~K}$ versus $3600 \mathrm{~K}$ ) than that adopted by Persi et al. (2007).

\subsubsection{V346 Norma}

This object has a luminosity of $\sim 135 L_{\odot}$ (Sandell \& Weintraub 2001), and was discovered in 1983 by Graham (1983) in the dark cloud Sa 187 in Norma. V346 Nor shows FUors-like characteristics (Reipurth 1985a; Graham \& Frogel 1985; Frogel \& Graham 1983), and is associated with a bipolar molecular outflow (Reipurth et al. 1997; Sandell \& Weintraub 2001). This protostar is located near the YSO Reipurth 13, consequently the outflow of V346 Nor may be affected by the presence of this other young star (Prusti et al. 1993).

In Figure 1.18 we present the observed SED of V346 Norma, which includes the spectra obtained with Spitzer-IRS in the 5-35 $\mu \mathrm{m}$ range (Green et al. 2006). The absorption around $10 \mu \mathrm{m}$ clearly seen in this spectrum is probably due to silicates. The observed fluxes have a relatively large scatter in the $10-100 \mu \mathrm{m}$ region, which is likely due to the different apertures used.

Table 4 lists the parameters corresponding to the SED model shown in Figure 1.18. This figure displays the SED model for three values of apertures: 60" (solid line), 30" (dotted-dashed line), and $11^{\prime \prime}$ (dashed line). The value obtained for the mass of the envelope, $M_{\text {env }}=0.3 M_{\odot}$, agrees with the $M_{\text {env }} \sim 0.5 M_{\odot}$ derived by Sandell \& Weintraub (2001) and is greater than the value estimated for the disk mass $\left(M_{\text {disk }}=0.05 M_{\odot}\right)$, however it has to be noted that the fit at $\mathrm{mm}$ wavelengths is somewhat poor.

\subsection{9. $\mathrm{OO} \mathrm{Ser}$}

OO Ser, previously known as DEOS (Serpens Deeply Embedded Outburst Star), is an embedded class I source (Enoch et al. 2009), located in the Serpens star-forming region at a distance of $311 \mathrm{pc}$ (de Lara et al. 1991). The outburst probably occurred in 1995 (Hodapp et al. 1996), however its nature or membership to the class I FUors is somewhat uncertain. After of the outburst, the source has been declining in brightness (Kóspál et al. 2007), and Hodapp et al. (2012) suggested that this tendency has already stopped. In Figure 1.19 we show the SED of OO Ser divided in two epochs, the outburst (1995-1996) period and the post-outburst stage, which was after 1996.

The fluxes cover the spectral range between $\sim 1-60 \mu \mathrm{m}$, with only two flux measurements available in the sub-mm range. Fluxes around $2 \mu \mathrm{m}$ have a relatively large dispersion. The parameters for the model in Figure 1.19 are listed in Table 4. The disk mass accretion rate value $\dot{M}_{\text {disk }}=5 \times 10^{-5} M_{\odot} \mathrm{yr}^{-1}$ is the highest of all class I FUors in our sample.

\subsubsection{0. $\operatorname{Re} 50 \mathrm{~N} I R S 1$}

Re 50 was discovered in the L1641 molecular cloud by Reipurth (1985b). Later observations of the source 
IRAS 05380-0728, located 1'.5 north of Re 50 (i.e., Re 50 N), allowed the identification of Re $50 \mathrm{~N}$ IRS1, which has a stellar counterpart observed at $3.6 \mu \mathrm{m}$ (Casali 1991). Re 50 N IRS1 is an embedded class I object, located at a distance of $460 \mathrm{pc}$ (Geers et al. 2009; Sandell \& Weintraub 2001) and associated with a bipolar molecular outflow (Reipurth \& Bally 1986). Strom \& Strom (1993) proposed Re 50 N IRS1 as a FU Orionis type object.

In Figure 1.20 we present the SED and model of Re $50 \mathrm{~N}$ IRS1. The observed fluxes obtained from the literature do not completely cover the range between the NIR and the $2000 \mu \mathrm{m}$. However, the ISO-SWS spectrum provides good coverage of the 5-15 $\mu \mathrm{m}$ range (Quanz et al. 2007b). The dispersion of the observed fluxes, both at $10 \mu \mathrm{m}$ and at longer wavelengths is relatively significant. In a first attempt to model these data we tested different sets of observations according to the period of time in which they were obtained. However, given the small number of fluxes available, this turned out to be inconvenient. Furthermore, because different apertures sizes were used in the flux determinations, this is likely the cause of the large dispersion.

Table 4 lists the parameters obtained from the model. The disk mass accretion rate $\dot{M}_{\text {disk }}=1.3 \times 10^{-6} M_{\odot} \mathrm{yr}^{-1}$ and the envelope mass accretion rate $\dot{M}=1.24 \times 10^{-5} M_{\odot} \mathrm{yr}^{-1}$ are higher than the values for class I protostars (Whitney et al. 2003a). However, $\dot{M}_{\text {disk }}$ is on the order of those obtained for the other class I FUors in our sample, which strengthens its classification as a FUor.

\subsubsection{V2492 Cygni}

This object, also known as PTF10NVG, IRAS 20496+4354, and VSXJ205126.1+440523, is a class I object located to the South-East of the Pelican North Nebula ( $d=550$ pc, Straizys et al. 1989; Bally \& Reipurth 2003; Covey et al. 2011), at an angular distance of about $2^{\circ}$ from the protostellar object and FUor HBC 722. Furthermore, V2492 Cyg may be associated with an outflow (Bally \& Reipurth 2003; Covey et al. 2011).

Covey et al. (2011) observed the outburst in 2010 while conducting a monitoring survey of the North American Nebula region, registering a brightness increase of $\sim 5$ mag in the optical and NIR. They found resemblances between this outburst and the one studied in 2004 by V1647 Ori, which, in turn, presents some characteristics similar to FUor events (see Section 3.1.8). Among these similarities are the weak P-Cygni profiles in the Balmer and Ca II lines (Covey et al. 2011; Aspin 2011b).

Moreover, V2492 Cyg has Na I D, K I, and He I blueshifted absorptions associated with strong outflows. However, V2492 Cygni does not show other typical characteristics of FUors, such as FG-supergiant spectral type in the optical and an M supergiant in the NIR (Covey et al. 2011).

Aspin (2011b) observed that the CO overtone bandheads are strongly in emission. They also noted that the properties of V2492 Cyg during its outburst are similar to the 2008 outburst of V1647 Ori, which in turn is more similar to an EX Lupi (the progenitor the EXor class) event than to a FUor event. In summary, whether V2492 Cygni is a FUor or an EXOR is still under debate (Kóspál 2011).

In Figure 1.21 we show the SEDs of V2492 Cyg corresponding to three epochs, before the 2010 outburst, and in two outburst periods, 2010 September and November. Pre-outburst fluxes (before 2010) cover the spectral range between $\sim 0.1-1300 \mu \mathrm{m}$, with fluxes around $80 \mu \mathrm{m}$ presenting a large dispersion. On the other hand, both SEDs for the 2010 September and November outbursts only cover the $\sim 0.1-3 \mu \mathrm{m}$ spectral range, which complicates the determination of reliable parameters for the external disk and envelope. The best models obtained for the different epochs reproduce satisfactorily the observed SEDs. The parameters for the models in the Figure 1.21 are listed in Table 4.

The derived parameters for the V2492 Cygni SED before the outburst agree with parameters for class I sources (Whitney et al. 2003a), and are also in concordance with the values obtained by Aspin (2011b) using the grid of Robitaille et al. (2006) and Robitaille et al. (2007). The disk mass accretion rate $\left(\dot{M}_{\text {disk }}=0.1 \times 10^{-6} M_{\odot} \mathrm{yr}^{-1}\right.$ versus $\left.0.4 \pm 0.5 \times 10^{-6} M_{\odot} \mathrm{yr}^{-1}\right)$, and the stellar parameters $\left(R_{*}=2.5 R_{\odot}\right.$ versus $2.8-3.0 R_{\odot}$ and $T_{*}=5000 \mathrm{~K}$ versus $6100-6500 \mathrm{~K}$ ) increase during the outburst event in a similar way as for other class I FU Orionis objects in our sample.

$$
\text { 3.2.12. V1331 Cygni }
$$

This protostar, also known as LkHa 120 and IRAS $20595+5009$, is located in the L988 complex at a distance of $\sim 550$ pc (see Herbig \& Dahm 2006 for a summary), and is associated with a bipolar molecular outflow (Levreault 1988; Mundt \& Eislöffel 1998). Biscaya et al. (1997) suggested the presence of a circumstellar disk with a mass of $\sim 0.5 M_{\odot}$ surrounded by a gaseous envelope. McMuldroch et al. (1993) observed in CO synthesis maps an external expanding gas ring, and Quanz et al. (2007a) detected two circumstellar rings of dust separated by a gap.

V1331 Cyg shares several characteristics with FUors, and it has been classified as a pre-outburst FUor (Welin 1976; Herbig 1989). However, its nature still remains uncertain (Biscaya et al. 1997; Sandell \& Weintraub 2001). Figure 1.22 shows the SED of V1331 Cyg. The fluxes obtained from the literature show a relatively large dispersion, which is likely due to the different periods in which they were obtained.

For this reason, the observed fluxes have been divided into two intervals of time. The first corresponds to the observations before 1991 and the second to the post-2001 period. The corresponding modeled SEDs are displayed with solid and dashed lines, respectively. Table 4 shows the values of the parameters corresponding to the SED defined by the data obtained before 1991. In brackets we list the values of the model parameters for the post-2001 data when they differ from the pre-1991 parameters. In our modeling we took into account the different spectral types corresponding to the two periods indicated, F0/F4 and G5, respectively (Chavarria-K. \& de Lara 1981; Hamann \& Persson 1992; Herbig et al. 2003). Using the spectral type calibration of Kenyon \& Hartmann (1995) we derived a temperature $T \sim 6600 \mathrm{~K}$ and $T \sim 5770 \mathrm{~K}$, respectively.

The two models have different disk masses $\left(\mathrm{M}_{\text {disk }}=0.1 M_{\odot}\right.$ versus $\left.0.02 M_{\odot}\right)$. In general, the parameter values for the second SED are lower, although this difference can only be considered marginal. One exception is the disk mass accretion rate, which decreased by an order of magnitude between 1991 and 2001 $\left(\dot{M}_{\text {disk }}=2.0 \times 10^{-6} M_{\odot} \mathrm{yr}^{-1}\right.$ versus $\left.0.1 \times 10^{-6} M_{\odot} \mathrm{yr}^{-1}\right)$. All this suggests that V1331 Cyg has entered into a post-outburst stage.

\subsubsection{3. $\mathrm{HBC} 722$}

HBC 722, also known as V2493 Cygni and PTF 10qpf, is an YSO located at $\sim 520 \mathrm{pc}$ in the North America/Pelican Nebula (e.g., Laugalys et al. 2006). The outburst of this star, which occurred 2010 March to August, was detected independently 
by Munari et al. (2010), from low resolution spectra, and by Semkov et al. (2010), from (BVRI) photometry and optical spectroscopy. Based on the similarity of the light curve with those of FU Ori and V1057 Cyg, Semkov et al. (2010) suggested that HBC 722 was a FUor-like object. More recently, this suggestion was confirmed by Miller et al. (2011) from infrared photometry and spectroscopy of this star, as well as high and low resolution optical spectroscopy. In particular, they reported an increase in the brightness of $4 \mathrm{mag}$, an optical spectrum consistent with a $\mathrm{G}$ supergiant and a NIR spectrum resembling those of late K-M giants/supergiants. Nevertheless, Kóspál et al. (2011) argued against the bona fide FUor classification of this source, based on its fast fading rate. Semkov et al. (2012), however, used the shape of the long-term light curve to confirm the FUor nature of HBC 722. Dunham et al. (2012) analyzed sub-mm continuum and molecular line emission indicating that HBC 722 is not associated with an outflow.

Figure 1.23 shows the pre-outburst and the post-outburst SEDs. The observed fluxes for both SEDs cover the spectral range from optical to $10 \mu \mathrm{m}$ particularly well. On the contrary, only a few data points are available for wavelengths around $100 \mu \mathrm{m}$ in the case of the post-outburst SED, whereas no data for $\lambda>10 \mu \mathrm{m}$ have been found for the pre-outburst SED. Fluxes for $\lambda<10 \mu \mathrm{m}$ allow a reliable identification of the outburst event, however the scarcity of fluxes above $10 \mu \mathrm{m}$, particularly in the pre-outburst SED, rendered uncertain the determination of the envelope parameters. Nevertheless, the best models obtained for the outburst SED (solid line, Figure 1.23) and the pre-outburst SED (dotted-dashed line) reproduce satisfactorily well the SEDs observed in the optical and infrared spectral regions.

Table 4 lists the parameters from the modeling of the postoutburst SED, and in brackets we indicate the values obtained for the pre-outburst SED when they differ. Notable differences are the stellar temperature $T_{*}$ and the disk mass accretion rate $\dot{M}_{\text {disk }} . T_{*}$ increases from $5600 \mathrm{~K}$ to $7100 \mathrm{~K}$, while $\dot{M}_{\text {disk }}$ increases from $0.4 \times 10^{-6} M_{\odot} \mathrm{yr}^{-1}$ to $4 \times 10^{-6} M_{\odot} \mathrm{yr}^{-1}$. Postoutburst parameters agree with previous estimates for other class I FU Orionis.

\subsubsection{Parsamian 21}

Par 21, also known as IRAS 19266+0932, was discovery by Parsamian (1965) and classified as a FUor by Staude \& Neckel (1992) based on an optical spectrum and infrared properties of the central star that illuminates a cometary nebula. These authors also associated this protostar with a small bipolar Herbig-Haro flow aligned along the polar axis of the nebula. Allen et al. (2004) classified the star as a class II object, and Kóspál et al. (2008) resolved a circumstellar envelope with a polar cavity and an edge-on disk on their high-resolution NIR direct and polarimetric images. However, Quanz et al. (2007b) suggest that this source is a post-asymptotic giant branch star, based on the detection of polycyclic aromatic hydrocarbon emission features on a 4-5 $\mu \mathrm{m}$ infrared spectrum obtained with Spitzer.

Figure 1.24 shows the observed SED for Par 21, including the 5-14 $\mu \mathrm{m}$ Spitzer-IRS spectrum (Quanz et al. 2007b). The model follows the shape of the Spitzer spectrum, but fails to reproduce individual fluxes at these wavelengths. In addition, the model underestimates the fluxes around $4 \mu \mathrm{m}$ by a factor of $\sim 3$ and overestimates the fluxes around $50 \mu \mathrm{m}$ by a factor of $\sim 10$. Consequently, the disk parameters, in particular the disk outer- or centrifugal radius $\left(R_{\mathrm{c}}\right)$ and the disk inner-radius $\left(R_{\min }\right)$, are likely to be poorly determined by our model. Table 4 presents the parameters corresponding to the model shown in Figure 1.24.
The distance to this source is uncertain (see Table 1), but for our SED model we adopted a distance of $1800 \mathrm{pc}$, as estimated by Sandell \& Weintraub (2001). The disk mass we derived $\left(M_{\text {disk }}=0.30 M_{\odot}\right)$ agrees with that determined by Sandell \& Weintraub (2001).

Kóspál et al. (2008) analyzed this source using a SED model that includes an optically thick and geometrically thin accretion disk with an optically thin envelope with no cavity. In addition, no central source is simulated, since during the outburst stage the inner disk contribution overwhelms the star flux (Hartmann \& Kenyon 1996). Moreover, they adopted an edge-on disk that obscures the central star. In general, the model parameters derived by Kóspál et al. (2008), including the envelope mass $\left(M_{\mathrm{env}}=0.22 M_{\odot}\right)$, roughly agree with our determinations. They also suggest that Par 21 has an edge-on disk that is consistent with our model inclination angle $\left(i=79^{\circ}\right)$. However, our inner disk radius is larger than what they derived.

More recently, Liu et al. (2011) analyzed the SEDs of a sample of Herbig Ae/Be stars, including Par 21, using the Robitaille et al. (2006) grid. They derive $M_{*}=3.74 M_{\odot}$, $R_{*}=5.68 R_{\odot}$, and $T_{*}=8511 \mathrm{~K}$. Both the stellar mass and radius are higher than our estimations (see Table 4), but we are in a good agreement on the stellar temperature. The inclination angle $i=87.13$ obtained by Liu et al. (2011) is consistent with our result of $i=79^{\circ}$. However, we find that our disk mass and disk mass accretion rate differ from their determinations. Their estimations of $M_{\text {disk }}=0.04 M_{\odot}$ and $\dot{M}_{\text {disk }}=4 \times 10^{-6} M_{\odot} \mathrm{yr}^{-1}$ correspond rather to a classical or inactive class II object than to an FUor. For the disk mass and mass accretion rate we derived $M_{\text {disk }}=0.3 M_{\odot}$ and $\dot{M}_{\text {disk }}=4 \times 10^{-6} M_{\odot} \mathrm{yr}^{-1}$, respectively, which is in good agreement with previous estimates for other class I FU Orionis.

\subsubsection{V2775 Ori}

This object, also known as 2MASS J05424848-0816347, was first reported by Caratti o Garatti et al. (2011) as a FUor in the L1641 region of the Orion molecular cloud, at a distance of 420 pc (Sandstrom et al. 2007; Menten et al. 2007; Kim et al. 2008). V2775 Ori is suspected to be part of a wide binary system with a separation of $\sim 17300 \mathrm{AU}(\sim 0.08 \mathrm{pc})$, associated to precessing jets (Caratti o Garatti et al. 2011). Fischer et al. (2012) observed this source in the near-IR and concluded that its spectra are consistent with a FU Orionis object. They observe $\mathrm{CO}$ absorption lines in the $K$-band, broad $\mathrm{H}_{2} \mathrm{O}$ absorption, strong and wide blueshifted $\mathrm{He} \mathrm{I}$, and a lack of atomic hydrogen emission.

In Figure 1.25 we show the SED of V2775 Ori divided in two epochs, the pre-outburst (before 2005) period and the post-outburst stage, after 2006. The outburst fluxes cover the $\sim 1-900 \mu \mathrm{m}$ spectral range, however the fluxes in the pre-outburst period only cover the spectral range between $\sim 1-70 \mu \mathrm{m}$, without any measurements available in the sub$\mathrm{mm}$ range. The best models obtained for the different epochs satisfactorily reproduce the observed SEDs. The parameters for the model shown in Figure 1.25 are listed in Table 4.

When comparing our results for the pre- and post-outburst SEDs, we see that the stellar temperature and the disk mass and mass accretion rate all increased during the outburst (see Table 4). In particular, $\dot{M}_{\text {disk }}$ increased by about two orders of magnitude from $0.6 \times 10^{-6} M_{\odot} \mathrm{yr}^{-1}$ to $10 \times 10^{-6} M_{\odot} \mathrm{yr}^{-1}$. On the other hand, the stellar mass, as well as the geometrical parameters $i=60^{\circ}$ and $\theta=7^{\circ}$, remain unchanged, while 
Table 5

Average Values for Class I and Class II FUors and YSOs

\begin{tabular}{|c|c|c|c|c|}
\hline Parameter & $\begin{array}{c}\text { Average Values }^{\mathrm{a}} \\
\text { Class I FUors }\end{array}$ & $\begin{array}{c}\text { Average Values } \\
\text { Class I YSOs }\end{array}$ & $\begin{array}{l}\text { Average Values }^{\mathrm{a}} \\
\text { Class II FUors }\end{array}$ & $\begin{array}{c}\text { Average Values } \\
\text { Class II YSOs }\end{array}$ \\
\hline$M_{*}\left(M_{\odot}\right)$ & 0.87 & 0.93 & 0.59 & 1.61 \\
\hline$T_{*}(\mathrm{~K})$ & 6077 & 3073 & 5775 & 4268 \\
\hline$\dot{M}\left(10^{-6} M_{\odot} \mathrm{yr}^{-1}\right)$ & 6.3 & 9.73 & 0.1 & 0.01 \\
\hline$R_{\mathrm{c}}(\mathrm{AU})$ & 124 & 397 & 157 & 239 \\
\hline$R_{\min }(\mathrm{AU})$ & 0.31 & 5.93 & 0.24 & 1.16 \\
\hline$M_{\text {disk }}\left(M_{\odot}\right)$ & 0.14 & 0.01 & 0.22 & 0.03 \\
\hline$\dot{M}_{\text {disk }}\left(10^{-6} M_{\odot} \mathrm{yr}^{-1}\right)$ & 8.3 & 0.6 & 9.1 & 0.2 \\
\hline
\end{tabular}

Notes.

a Averages of the results shown in the Tables 3 and 4 for class I and class II, respectively.

b Averages values from Robitaille et al. (2007).

the envelope mass accretion rate decreases by one order of magnitude from $3 \times 10^{-6} M_{\odot} \mathrm{yr}^{-1}$ to $7 \times 10^{-7} M_{\odot} \mathrm{yr}^{-1}$.

Caratti o Garatti et al. (2011) find that this source is one of the lowest mass YSOs presenting a strong outburst. Based on the features of its spectra (strong $\mathrm{CO}$ bandheads, $\mathrm{H}_{2} \mathrm{O}$ broadband absorption, $\mathrm{Br} \gamma$ in emission) they adopted an $\mathrm{M}$ spectral type. In comparisons of their NIR spectra with AMESDUSTY models (Allard et al. 2001) they find a reasonable match for $T_{\text {eff }} \sim 3200 \mathrm{~K}$. Furthermore, applying the main-sequence models of Siess et al. (2000) they derived a stellar mass of $\sim 0.24 M_{\odot}$.

Fischer et al. (2012) modeled this source and obtained that the disk accretion rate increased in around one order of magnitude $\left(\sim 2 \times 10^{-6} M_{\odot} \mathrm{yr}^{-1}\right.$ to $\left.\sim 10^{-5} M_{\odot} \mathrm{yr}^{-1}\right)$ and that the envelope mass accretion rate remained constant with a value of $7 \times 10^{-7} M_{\odot} \mathrm{yr}^{-1}$ and suggested that V2775 Ori is approaching the end of the envelope dominated phase. For their best model they adopted $R_{*}=2.09 R_{\odot}, T=4000 \mathrm{~K}$ and $M_{*}=0.5 M_{\odot}$, and with an outer radius of $10000 \mathrm{AU}$ they derived an envelope mass of $0.09 M_{\odot}$, an opening angle of $25^{\circ}$, and an inclination of $49^{\circ}$.

From our models we obtain $M_{*}=0.5 M_{\odot}$, which is in good agreement with Caratti o Garatti et al. (2011). However, the stellar temperature estimated before and after the outburst (5600 $\mathrm{K}$ and $6800 \mathrm{~K}$, respectively) is significantly higher than what Caratti o Garatti et al. (2011) and Fischer et al. (2012) obtained. Geometrical parameters as the opening angle and inclination are similar, although somewhat higher than the those derived by Fischer et al. (2012). On the other hand, the increase in the disk mass-accretion rate we obtain is one order of magnitude larger than what they derive.

Based on the increase of its envelope mass-accretion rate from $3 \times 10^{-6} M_{\odot} \mathrm{yr}^{-1}$ to $7 \times 10^{-7} M_{\odot} \mathrm{yr}^{-1}$ during the outburst, we suggest that before the outburst this was a Class I object, but now during the outburst it is in the late stages of that class. This is in agreement with Fischer et al. (2012).

\section{RESULTS}

In this section we analyze the results derived from the modeling of the 24 known and candidate FUors in our sample. We compare parameter values for these objects with those of standard class II and class I objects that are not in an eruptive phase, which are obtained from the sample of YSOs in Taurus modeled by Robitaille et al. (2007). Table 5 lists the average values for each parameter of the class II and class I FUors. For sources RNO 1B, V1647 Ori, OO Ser, V2492 Cyg, HBC 722, V2775 Ori, and V1331 Cyg we obtained more than one solution, corresponding to different periods of observations. In those cases, we choose the parameters corresponding to the outburst stage for computing the average. Table 5 also gives average values of these parameters for standard class II and class I YSOs from Robitaille et al. (2007). From Table 5 we see that class II and class I FUors disks have smaller inner radii $\left(R_{\min }\right)$ in comparison with the average values for the standard class II and class I from Robitaille et al. (2007). In addition, for FUors the centrifugal radii of the circumstellar disks are also smaller than for classical YSOs.

To better describe and compare how the different model parameters change between the FUors during the outburst and the standard class II and class I objects in quiescence stage, we analyze the cumulative distribution of the disk mass, the disk mass accretion rate, the envelope mass accretion rate, and the stellar temperature. These parameters were selected because they show the largest variations. For the typical class II and class I objects, we use the parameters from the models of Robitaille et al. (2007) for standard YSOs in Taurus. The resulting distributions are shown in Figure 2. We apply the Kolmogorov-Smirnov (K-S) test to compare these distributions for each of the four parameters selected. Table 6 lists the median values for both groups, as well as the K-S test results (i.e., the maximum difference $D$ between the distributions and the significance or confidence level $s$ ). The cumulative distributions for both groups (standard class II and class I objects in Taurus and FUors) are different with a high level of confidence. ${ }^{9}$ We also see that within each parameter, the values for each group are distributed in different ways.

On average, FUors disks are more massive and have higher accretion rates than standard class II and class I disks. None of the disks in standard class II and class I objects have masses above $0.06 M_{\odot}$, whereas $\sim 80 \%$ of the disks in FUors have masses $\geqslant 0.10 M_{\odot}$. Nevertheless, the mass distributions for classical YSOs and FUors have similar spreads. Standard class II and class I objects span at least two orders of magnitude in mass from $2.5 \times 10^{-4} M_{\odot}$ to $6 \times 10^{-2} M_{\odot}$ (see also Andrews \& Williams 2005), whereas FUors disks span a mass range from $0.01 M_{\odot}$ to $0.37 M_{\odot}$. The values listed on Table 5 indicate that class II and class I FUors show increases in the disk mass by one order of magnitude (see also Table 6).

Regarding the disk mass accretion rate, $\sim 90 \%$ of the FUors have $\dot{M}_{\text {disk }}>10^{-6} M_{\odot} \mathrm{yr}^{-1}$, while $\sim 95 \%$ of the standard class II and class I objects have $\dot{M}_{\text {disk }}<10^{-6} M_{\odot} \mathrm{yr}^{-1}$. The

\footnotetext{
$\overline{9}$ If both distributions were identical, then $s=1$.
} 

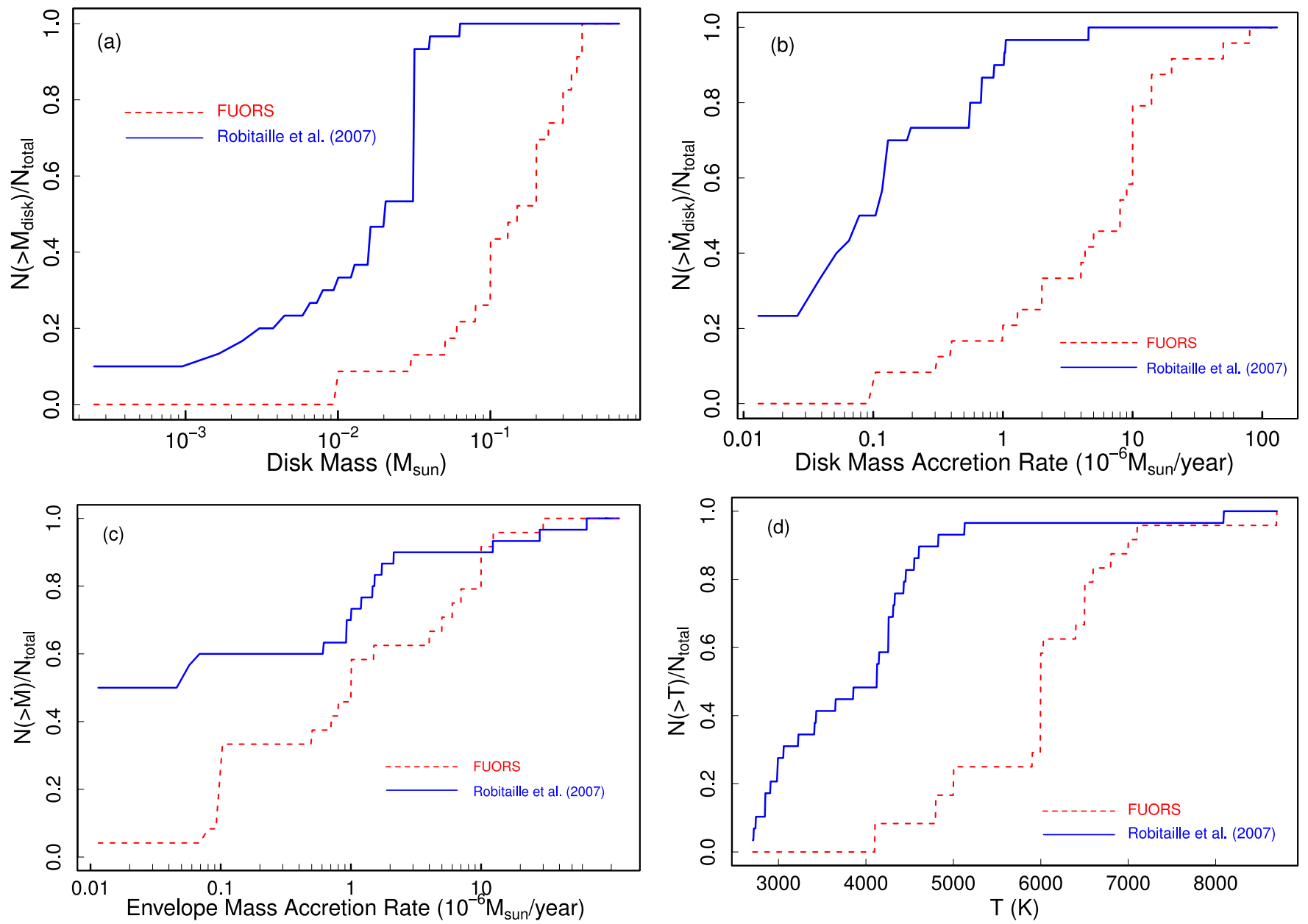

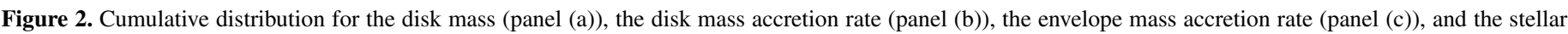

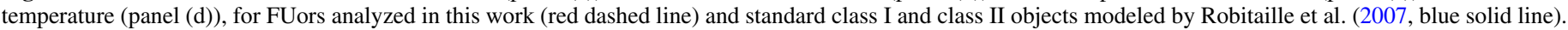

(A color version of this figure is available in the online journal.)

Table 6

Median Values of FUors and Classical T Tauri Stars and K-S Analysis

\begin{tabular}{lcccc}
\hline \hline Parameter & $\begin{array}{c}\text { Classes I and II } \\
\text { FUors }\end{array}$ & $\begin{array}{c}\text { Classes I and II from } \\
\text { Robitaille et al. (2007) }\end{array}$ & $D^{\mathrm{a}}$ & $s^{\mathrm{b}}$ \\
\hline$\dot{M}_{\text {disk }}\left(10^{-6} M_{\odot} \mathrm{yr}^{-1}\right)$ & 8.0 & 0.1 & 0.79 & $3 \times 10^{-8}$ \\
$M_{\text {disk }}\left(M_{\odot}\right)$ & 0.15 & 0.02 & 0.83 & $3 \times 10^{-9}$ \\
$\dot{M}\left(10^{-6} M_{\odot} \mathrm{yr}^{-1}\right)$ & 1.00 & 0.03 & 0.84 & $4 \times 10^{-8}$ \\
$T_{*}(\mathrm{~K})$ & 6000 & 3989 & 0.84 & $4 \times 10^{-9}$ \\
\hline
\end{tabular}

Notes.

${ }^{a} D$ : maximum difference.

b $s$ : significance or confidence level.

median mass accretion rate for FUors is $\sim 10^{-5} M_{\odot} \mathrm{yr}^{-1}$, in contrast with $\sim 10^{-7} M_{\odot} \mathrm{yr}^{-1}$ for classical YSOs (see Table 6). Despite being unusual or rare, a significant amount of mass can be accumulated onto the central star during relatively short periods of time (the FUor events), which contributes to its final mass.

A comparison of the behavior of the envelope mass accretion rate for both distributions shows that a large fraction of FUors $(\sim 70 \%)$ have accretion rates $>10^{-7} M_{\odot} \mathrm{yr}^{-1}$. In contrast, $60 \%$ of the classical YSOs have accretion rates below this value. On average, FUors have higher envelope mass accretion rates than standard class II and class I sources $\left(\sim 10^{-6} M_{\odot} \mathrm{yr}^{-1}\right.$ versus $\sim 10^{-8} M_{\odot} \mathrm{yr}^{-1}$, respectively, see Table 6). For class I FUors, the envelope mass accretion rate remains practically unchanged during the FU Orionis stage (see Table 5). In the cases where we had an SED before and after the outburst, this parameter remained unchanged for both SED models (see Tables 3 and 4). Figure 3 shows the envelope mass accretion rate, analyzed per YSO class, that is, for the class I FUors sample versus standard class I objects (left panel) and for the class II FUors group versus classical class II YSOs (right panel). The distributions of class I FUors and standard class I objects are similar $(s=0.15$, $D=0.31$ ), whereas class II FUors and classical class II are different $\left(s=1.5 \times 10^{-4}, D=0.89\right)$. 

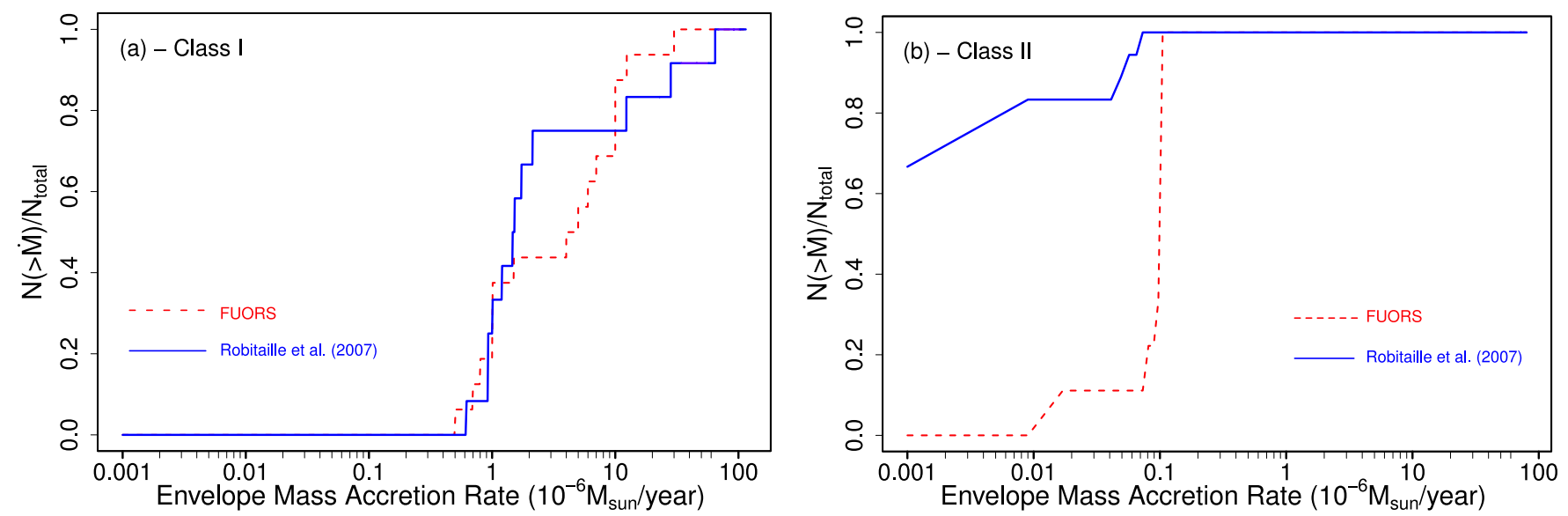

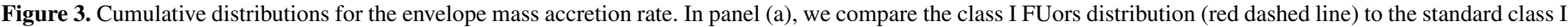

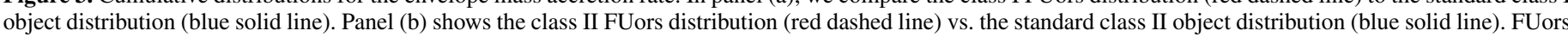
have been analyzed in this contribution. Standard class I and class II objects have been modeled by Robitaille et al. (2007).

(A color version of this figure is available in the online journal.)

The case for the stellar temperature is different (see Figure 2, bottom right panel). The distributions for both groups are similar in shape, only shifted by about $2000 \mathrm{~K}$ to higher temperatures for class II and class I FUors, which reflects the observed rise in stellar luminosity during the outburst event. The higher stellar temperature would also account for the hotter or earlier spectral type.

For seven of the stars in our sample, two class II (RNO 1B, V1647 Ori) and five class I (OO Ser, V2492 Cyg, HBC 722, V2775 Ori, V1313 Cyg), we modeled two SEDs (see Tables 3 and 4) during the outburst and at the quiescence stage. Envelope parameters such as mass, radius, and mass accretion rate do change. This suggests that the outbursts are triggered by an instability after a long build-up phase. In general, the remaining disk and stellar parameters change significantly.

\section{SUMMARY AND DISCUSSION}

In this work we present the modeling of the SEDs of a sample of 24 class II and class I FU Orionis stars. These SEDs were constructed from fluxes obtained from the literature (Table 2), including Spitzer-IRS infrared spectra in the 5-35 $\mu \mathrm{m}$ range for V1515 Cyg, BBW 76, FU Ori, V346 Nor and V1057 Cyg, and in the 5-14 $\mu \mathrm{m}$ range for RNO 1B, RNO 1C, L1551 IRS5, and Par 21 (Green et al. 2006; Quanz et al. 2007b). For Re $50 \mathrm{~N}$ IRS1, we used an ISO-SWS spectrum in the 5-15 $\mu \mathrm{m}$ range obtained by Quanz et al. (2007b).

Initially we modeled each source by applying the grid of Robitaille et al. (2006), to later use these models as starting points for a more refined analysis using the code of Whitney et al. (2003a). The parameters corresponding to the best model fits are given in Tables 3 and 4 for class II and class I FUors, respectively. Figures 1.1 to 1.25 show the corresponding SEDs. For sources V1515 Cyg, BBW 76, PP 13S, V1647 Ori, FU Ori, V1057 Cyg, Z CMa, L1551 IRS5, ISO-Cha 192, V2492 Cyg, V1331 Cyg, Par 21, and V2775 Ori we compared our parameter values with those derived by other authors, finding in general a good agreement. For the remaining 11 sources, this is the first time a model of their SED was derived.

Figure 2 shows the cumulative distribution functions of disk masses, disk accretion rates, envelope accretion rates, and stellar temperatures of FUors in our sample and standard class II and class I objects in a quiescence state from Robitaille et al. (2007).
Table 6 gives the median values for both groups. The comparison shows that:

1. On average FUors disks are more massive than standard class II and class I object disks. About $80 \%$ of FUors disks have masses $>0.1 M_{\odot}$, while standard class II and class I objects have disk masses $<0.06 M_{\odot}$.

2. Disks mass accretion rates are higher for FUors than for classical YSOs. The great majority of FUors ( $90 \%)$ have $\dot{M}_{\text {disk }}>10^{-6} M_{\odot} \mathrm{yr}^{-1}$, whereas $\sim 95 \%$ of the standard class II and class I objects have $\dot{M}_{\text {disk }}<10^{-6} M_{\odot} \mathrm{yr}^{-1}$. Median disk accretion rates are $\sim 10^{-5} M_{\odot} \mathrm{yr}^{-1}$ versus $\sim 10^{-7} M_{\odot} \mathrm{yr}^{-1}$ for FUors and classical YSOs, respectively.

3. The distributions of envelope accretion rates for class I FUors and standard class I objects are indistinguishable. Most FUors $(\sim 70 \%)$ have envelope accretion rates $>10^{-7} M_{\odot} \mathrm{yr}^{-1}$. Median envelope accretion rates are $\sim 10^{-6} M_{\odot} \mathrm{yr}^{-1}$ versus $\sim 10^{-8} M_{\odot} \mathrm{yr}^{-1}$ for FUors and standard YSOs, respectively.

4. The distribution of stellar temperatures for FUors and classical YSOs are similar in shape, but the FUors are shifted $\sim 2000 \mathrm{~K}$ to higher temperatures.

The cumulative distributions for confirmed and candidate FUors (see Table 1) show no significant differences, suggesting that most candidate objects, in fact, belong to the FUor class. We caution, however, on the small number of objects in each class (14 confirmed and 10 candidate FUors).

For the seven objects in our sample for which we have SEDs both in the outburst and in the quiescence stage (see Tables 3 and 4), two class II (RNO 1B, and V1647 Ori) and five class I (OO Ser, V1313 Cyg, V2492 Cyg, HBC 722, and V2775 Ori), we note that while the disk and stellar parameters show variations, the envelope parameters $\left(\dot{M}, R_{\max }\right.$, and $\left.M_{\text {env }}\right)$ do not change, suggesting the outbursts are triggered by an instability after a long build-up phase.

The current scenario of FUor events states that the circumstellar disk of a YSO builds up material injected from the envelope until it becomes thermally (Frank et al. 1992; Bell \& Lin 1994; Hartmann \& Kenyon 1996) and/or gravitationally (Zhu et al. 2009, 2010; Vorobyov \& Basu 2005, 2006, 2010) unstable. In particular, using the model parameters and disk properties listed in Tables 3 and 4 we calculated the Toomre $Q$ gravitational stability parameter (Toomre 1964). If $Q<1$, the disk 
is unstable. Most of the models are unstable for $R>5-20$ AU. The only exception is L 1551 IRS 5, which is gravitationally unstable at a larger scale $(R \gtrsim 50 \mathrm{AU})$. Nevertheless, all disks are unstable well inside the centrifugal radius (see Table 5). Consequently, gravitational instabilities may contribute to the outburst eruptions, in addition to thermal instabilities, resulting in an increase of the mass accretion onto the central object. What we have described so far agrees with this picture. However, the disk mass accretion rate $\dot{M}_{\text {disk }} \sim 10^{-5} M_{\odot} \mathrm{yr}^{-1}$ we obtain (see Table 6) is one order of magnitude lower than the $\sim 10^{-4} M_{\odot} \mathrm{yr}^{-1}$ predicted by the theory (Frank et al. 1992; Hartmann \& Kenyon 1996). Nevertheless, previous models of individual FU Orionis objects obtain $\dot{M}_{\text {disk }}$ values consistent with those presented in this work (see, e.g., Pfalzner 2008; Aspin et al. 2008).

Although the average values for the parameters for both groups of FUors are similar to those theoretically expected, the individual values listed for each object in Tables 3 and 4 differ significantly. This can be attributed in part to the fact that the group of the FUors itself is not a homogeneous sample. While they all share a particular set of characteristics, they appear in different ways for each object. For instance, while all FUors show a sudden brightness increase of several magnitudes, followed by a slow decrease to their previous state, the way the brightness jump develops in time is different for each object. A clear example of this is the great diversity in the light curves of the three prototypes of the class (Hartmann \& Kenyon 1996). It is therefore reasonable to expect that the values of individual parameters of each member of the group will ultimately differ.

Lastly, we would like to draw attention to three sources in particular. V1647 Ori is a special case on the FUors sample because it has been well studied before and after the outburst, having SEDs for both epochs. This makes that source a prime candidate for the study of the FU Orionis event, although it has to be approached with care, since its classification as a FUor or EXOR is still under debate (Aspin et al. 2006; Aspin 2011a; Semkov \& Peneva 2012). The other two particular sources are V2492 Cyg and HBC 722. These objects are, at the moment of writing, the last two for which a FUor-like outburst has been observed. They show the proper characteristics proper for a bona fide FUor, as shown in Sections 3.2.11 and 3.2.13, however their inclusion in the FUor class is still not certain. Nevertheless, our SED modeling shows behaviors similar to V1647 Ori, the former newest member of the class.

V1647 Ori and V2492 Cyg also show EXOR characteristics, and from our modeling we see that they do not show a large variation between the outburst and the quiescent phases. For example, their disk masses do not change with the outburst (see Tables 3 and 4), and the variation of the disk mass accretion rate is lower than for other FUors. Nevertheless, when compared with other FUors, the parameter values derived for those sources are still within the range established by the rest of the FUors sample, and could then be considered FUors. However, if we had just analyzed only those two sources while taking into consideration that EXOR outbursts are thought to be "scaled-down" versions of FUor outbursts, it is very likely that they would have been considered EXORS. This shows the uncertainty and difficulty of disentangling the two types of outburst episodes.

Despite sharing common properties, each FUor or FUor candidate has its own peculiarities that are not currently well understood. It is therefore of great interest to study the most extreme objects of the class to reach a full understanding of this period of great activity in circumstellar disks.
The work we have presented here is the first compilation of SEDs of the currently known FUors. Of the 26 currently known FUors, two do not have enough observations to construct the SEDs, and thus are not analyzed. For 21 of the remaining 24 we compiled the observations taken in all wavelengths, producing the most complete SEDs possible so far. For three FUors (AR 6A, AR 6B, V2492 Cyg), SEDs for only a limited range $(\lambda<20 \mu \mathrm{m})$ were constructed and thus values for the derived parameters are not fully determined. Finally, for 11 of the 24 FUors analyzed (V1735 Cyg, V883 Ori, RNO 1B, RNO 1C, AR 6A, AR 6B, V900 Mon, V346 Nor, OO Ser, RE 50 N IRS 1, and HBC 722) we provide for the first time a complete SED modeling to determine the physical and geometrical parameters of the star+disk+envelope system. Furthermore, this is the first time all known FUors with an observed SED are modeled with the same code at the same time, providing a homogeneous set of results. The data we present here will be of great help for future studies in the field.

We thank Dr. Barbara A. Whitney for thoroughly reading the manuscript and for providing comments and suggestions that greatly improved and clarified the content of the paper. We are also grateful to Dr. Sascha P. Quanz for sending us Spitzer-IRS and ISO-SWS spectra and to Dr. Joel D. Green for providing guide to access to Spitzer-IRS spectra. We appreciate the careful revision of the paper done by the referee as well as his/her suggestions, which improved the content and the presentation of this work.

\section{REFERENCES}

Ábrahám, P., Kóspál, Á., Csizmadia, S., et al. 2004a, A\&A, 428, 89 Ábrahám, P., Kóspál, Á., Csizmadia, S., et al. 2004b, A\&A, 419, L39 Acosta-Pulido, J. A., Kun, M., Ábrahám, P., et al. 2007, AJ, 133, 2020 Adams, F. C., Lada, C. J., \& Shu, F. H. 1987, ApJ, 312, 788

Allard, F., Hauschildt, P. H., Alexander, D. R., Tamanai, A., \& Schweitzer, A. 2001, ApJ, 556, 357

Allen, L. E., Calvet, N., D’Alessio, P., et al. 2004, ApJS, 154, 363

Alonso-Albi, T., Fuente, A., Bachiller, R., et al. 2009, A\&A, 497, 117

Andrews, S. M., Rothberg, B., \& Simon, T. 2004, ApJL, 610, L45

Andrews, S. M., \& Williams, J. P. 2005, ApJ, 631, 1134

Aspin, C. 2011a, AJ, 142, 135

Aspin, C. 2011b, AJ, 141, 196

Aspin, C., Barbieri, C., Boschi, F., et al. 2006, AJ, 132, 1298

Aspin, C., Beck, T. L., \& Reipurth, B. 2008, AJ, 135, 423

Aspin, C., \& Reipurth, B. 2000, MNRAS, 311, 522

Aspin, C., \& Reipurth, B. 2003, AJ, 126, 2936

Aspin, C., Reipurth, B., Beck, T. L., et al. 2009, ApJL, 692, L67

Aspin, C., Reipurth, B., Herczeg, G. J., \& Capak, P. 2010, ApJL, 719, L50

Aspin, C., \& Sandell, G. 2001, MNRAS, 328, 751

Bally, J., \& Reipurth, B. 2003, AJ, 126, 893

Beichman, C., \& Harris, S. 1981, ApJ, 245, 589

Bell, K. R., \& Lin, D. N. C. 1994, ApJ, 427, 987

Biscaya, A. M., Rieke, G. H., Narayanan, G., Luhman, K. L., \& Young, E. T. 1997, ApJ, 491, 359

Briceño, C., Vivas, A. K., Hernández, J., et al. 2004, ApJL, 606, L123

Calvet, N. 1998, in AIP Conf. Proc. 431, Accretion Processes in Astrophysical Systems: Some Like It Hot!, ed. S. S. Holt \& T. R. Kallman (Melville, NY: AIP), 495

Calvet, N., Hartmann, L., \& Kenyon, S. J. 1991, ApJ, 383, 752

Cambresy, L., Copet, E., Epchtein, N., et al. 1998, A\&A, 338, 977

Canovas, H., Min, M., Jeffers, S. V., Rodenhuis, M., \& Keller, C. U. 2012, A\&A, 543,70

Caratti o Garatti, A., Garcia Lopez, R., Scholz, A., et al. 2011, A\&A, 526, L1

Carr, J. S. 1989, ApJ, 345, 522

Carr, J. S. 1990, AJ, 100, 1244

Carr, J. S., Harvey, P. M., \& Lester, D. F. 1987, ApJL, 321, L71

Casali, M. M. 1991, MNRAS, 248, 229

Chandler, C. J., \& Richer, J. S. 2000, ApJ, 530, 851

Chavarria, C. 1981, A\&A, 101, 105 
Chavarria-K., C., \& de Lara, E. 1981, RMxAA, 6, 159

Clarke, A. J., Lodato, G., Melnikov, S. Y., \& Ibrahimov, M. A. 2005a, MNRAS, 361,942

Clarke, A. J., Oudmaijer, R. D., \& Lumsden, S. L. 2005b, MNRAS, 363, 1111 Cohen, M., Aitken, D. K., Roche, P. F., \& Williams, P. M. 1983, ApJ, 273, 624 Cohen, M., \& Barlow, M. J. 1974, ApJ, 193, 401

Cohen, M., Harvey, P. M., Wilking, B. A., \& Schwartz, R. D. 1984, ApJ, 278, 671

Cohen, M., Hollenbach, D. J., Haas, M. R., \& Erickson, E. F. 1988, ApJ, 329,863

Cohen, M., \& Kuhi, L. V. 1979, ApJS, 41, 743

Cohen, M., \& Schwartz, R. D. 1983, ApJ, 265, 877

Cohen, M., \& Schwartz, R. D. 1987, ApJ, 316, 311

Connelley, M. S., Reipurth, B., \& Tokunaga, A. T. 2007, AJ, 133, 1528

Covey, K. R., Hillenbrand, L. A., Miller, A. A., et al. 2011, AJ, 141, 40

Davidson, J. A., \& Jaffe, D. T. 1984, ApJL, 277, L13

Davis, C. J., Mundt, R., Eislöffel, J., \& Ray, T. P. 1995, Ap\&SS, 233, 51

de Lara, E., Chavarria-K., C., \& Lopez-Molina, G. 1991, A\&A, 243, 139

Dent, W. R. F., Matthews, H. E., \& Ward-Thompson, D. 1998, MNRAS, 301, 1049

Devine, D., Reipurth, B., \& Bally, J. 1999, AJ, 118, 972

Doppmann, G. W., Greene, T. P., Covey, K. R., \& Lada, C. J. 2005, AJ, 130,1145

Dunham, M. M., Arce, H. G., Bourke, T. L., et al. 2012, ApJ, 755, 157

Egan, M. P., Price, S. D., Kraemer, K. E., et al. 2003, yCat, 5114, 0

Egan, M. P., Price, S. D., Shipman, R. F., et al. 1999, in ASP Conf. Ser. 177, Astrophysics with Infrared Surveys: A Prelude to SIRTF, ed. M. D. Bicay, R. M. Cutri, \& B. F. Madore (San Francisco, CA: ASP), 404

Eisloeffel, J., Hessman, F. V., \& Mundt, R. 1990, A\&A, 232, 70

Elias, J. H. 1978, ApJ, 223, 859

Enoch, M. L., Evans, N. J., II, Sargent, A. I., \& Glenn, J. 2009, ApJ, 692, 973

Evans, N. J., II, Balkum, S., Levreault, R. M., Hartmann, L., \& Kenyon, S. 1994, ApJ, 424, 793

Evans, N. J., II, Dunham, M. M., Jørgensen, J. K., et al. 2009, ApJS, 181, 321

Fazio, G. G., Hora, J. L., Allen, L. E., et al. 2004, ApJS, 154, 10

Fernandez, M. 1995, A\&AS, 113, 473

Fischer, W. J., Megeath, S. T., Tobin, J. J., et al. 2012, ApJ, 756, 99

Frank, J., King, A., \& Raine, D. 1992, Accretion Power in Astrophysics (2nd ed.; Cambridge: Cambridge Univ. Press)

Fridlund, C. V. M., Nordh, H. L., van Duinen, R. J., Aalders, J. W. G., \& Sargent, A. I. 1980, A\&A, 91, L1

Frogel, J. A., \& Graham, J. A. 1983, IAUC, 3792, 2

Geers, V. C., van Dishoeck, E. F., Pontoppidan, K. M., et al. 2009, A\&A, 495,837

Gómez, M., \& Mardones, D. 2003, AJ, 125, 2134

Gómez, M., Persi, P., Marenzi, A. R., Roth, M., \& Tapia, M. 2004, A\&A, 423, 629

Goodrich, R. W. 1987, PASP, 99, 116

Graham, J. A. 1983, IAUC, 3785, 1

Graham, J. A., \& Frogel, J. A. 1985, ApJ, 289, 331

Gramajo, L. V., Whitney, B. A., Kenyon, S. J., Gómez, M., \& Merrill, K. M. 2007, AJ, 133, 1911

Grankin, K. N., \& Artemenko, S. A. 2009, IBVS, 5905, 1

Gredel, R. 1994, A\&A, 292, 580

Green, J. D., Evans, N. J., II, Kóspál, Á., et al. 2011, ApJL, 731, L25

Green, J. D., Hartmann, L., Calvet, N., et al. 2006, ApJ, 648, 1099

Greene, T. P., \& Lada, C. J. 1996, AJ, 112, 2184

Gregorio-Hetem, J. 2008, in Handbook of Star Forming Regions: Vol. II, The Southern Sky, ed. B. Reipurth (San Francisco, CA: ASP), 1

Guieu, S., Rebull, L. M., Stauffer, J. R., et al. 2009, ApJ, 697, 787

Hamann, F., \& Persson, S. E. 1992, ApJ, 394, 628

Hartmann, L., \& Calvet, N. 1995, AJ, 109, 1846

Hartmann, L., Hinkle, K., \& Calvet, N. 2004, ApJ, 609, 906

Hartmann, L., \& Kenyon, S. J. 1985, ApJ, 299, 462

Hartmann, L., \& Kenyon, S. J. 1987a, ApJ, 312, 243

Hartmann, L., \& Kenyon, S. J. 1987b, ApJ, 322, 393

Hartmann, L., \& Kenyon, S. J. 1996, ARA\&A, 34, 207

Hartmann, L., Kenyon, S. J., Hewett, R., et al. 1989, ApJ, 338, 1001

Harvey, P. M., Huard, T. L., Jørgensen, J. K., et al. 2008, ApJ, 680, 495

Harvey, P. M., \& Wilking, B. A. 1982, PASP, 94, 285

Henning, T., Burkert, A., Launhardt, R., Leinert, Ch., \& Stecklum, B. 1998, A\&A, 336, 565

Herbig, G. H. 1977, ApJ, 217, 693

Herbig, G. H. 1989, in ESO Workshop on Low Mass Star Formation and Premain Sequence Objects, ed. B. Reipurth (Garching: ESO), 233

Herbig, G. H. 2008, AJ, 135, 637

Herbig, G. H., \& Dahm, S. E. 2006, AJ, 131, 1530
Herbig, G. H., Petrov, P. P., \& Duemmler, R. 2003, ApJ, 595, 384

Herbst, W., Racine, R., \& Warner, J. W. 1978, ApJ, 223, 471

Hessman, F. V., Eisloeffel, J., Mundt, R., et al. 1991, ApJ, 370, 384

Heyer, M. H., Ladd, E. F., Myers, P. C., \& Campbell, B. 1990, AJ, 99, 1585

Hillenbrand, L. A., Strom, S. E., Vrba, F. J., \& Keene, J. 1992, ApJ, 397, 613

Hilton, J., \& Lahulla, J. F. 1995, A\&AS, 113, 325

Hodapp, K.-W., Capps, R. W., Strom, S. E., Salas, L., \& Grasdalen, G. L. 1988, ApJ, 335, 814

Hodapp, K. W., Chini, R., Watermann, R., \& Lemke, R. 2012, ApJ, 744, 56

Hodapp, K.-W., Hora, J. L., Rayner, J. T., Pickles, A. J., \& Ladd, E. F. 1996, ApJ, 468,861

Hogerheijde, M. R., van Dishoeck, E. F., Blake, G. A., \& van Langevelde, H. J. 1997, ApJ, 489, 293

Ibrahimov, M. A. 1999, IBVS, 4691, 1

Ishihara, D., Onaka, T., Kataza, H., et al. 2010, A\&A, 514, A1

Itagaki, K., Nakano, S., \& Yamaoka, H. 2008, IAUC, 8968, 2

Kaas, A. A., Olofsson, G., Bontemps, S., et al. 2004, A\&A, 421, 623

Keene, J., \& Masson, C. R. 1990, ApJ, 355, 635

Kenyon, S. J., Calvet, N., \& Hartmann, L. 1993a, ApJ, 414, 676

Kenyon, S. J., \& Hartmann, L. 1989, ApJ, 342, 1134

Kenyon, S. J., \& Hartmann, L. 1995, ApJS, 101, 117

Kenyon, S. J., Hartmann, L., Gomez, M., Carr, J. S., \& Tokunaga, A. 1993b, AJ, 105,1505

Kenyon, S. J., Hartmann, L., \& Hewett, R. 1988, ApJ, 325, 231

Kenyon, S. J., Hartmann, L., Imhoff, C. L., \& Cassatella, A. 1989, ApJ, 344,925

Kenyon, S. J., \& Hartmann, L. W. 1988, BAAS, 20, 1090

Kenyon, S. J., \& Hartmann, L. W. 1991, ApJ, 383, 664

Kenyon, S. J., Kolotilov, E. A., Ibragimov, M. A., \& Mattei, J. A. 2000, ApJ, 531, 1028

Kim, M. K., Hirota, T., Honma, M., et al. 2008, PASJ, 60, 991

Kolotilov, E. A., \& Petrov, P. P. 1983, PAZh, 9, 171

Koresko, C. D., Beckwith, S. V. W., Ghez, A. M., Matthews, K., \& Neugebauer, G. 1991, AJ, 102, 2073

Kóspál, Á. 2011, A\&A, 535, 125

Kóspál, Á., Ábrahám, P., Acosta-Pulido, J. A., et al. 2011, A\&A, 527, A133

Kóspál, Á., Ábrahám, P., Apai, D., et al. 2008, MNRAS, 383, 1015

Kóspál, Á., Ábrahám, P., Csizmadia, S., et al. 2004, PADEU, 14, 119

Kóspál, Á., Ábrahám, P., Prusti, T., et al. 2007, A\&A, 470, 211

Kun, M. 2008, IBVS, 5850, 1

Lachaume, R. 2004, A\&A, 422, 171

Lada, C. J. 1987, in IAU Symp. 115, Star Forming Regions, ed. M. Peimbert \& J. Jugaku (Cambridge: Cambridge Univ. Press), 1

Ladd, E. F., Fuller, G. A., Padman, R., Myers, P. C., \& Adams, F. C. 1995, ApJ, 439,771

Laugalys, V., Straižys, V., Vrba, F. J., et al. 2006, BaltA, 15, 483

Lawrence, A., Warren, S. J., Almaini, O., et al. 2007, MNRAS, 379, 1599

Lay, O. P., Carlstrom, J. E., Hills, R. E., \& Phillips, T. G. 1994, ApJL, 434, L75

Lee, C.-F., Mundy, L. G., Stone, J. M., \& Ostriker, E. C. 2002, ApJ, 576, 294

Leinert, C., Richichi, A., \& Haas, M. 1997, A\&A, 318, 472

Levreault, R. M. 1983, ApJ, 265, 855

Levreault, R. M. 1988, ApJS, 67, 283

Li, W., Evans, N. J., II, Harvey, P. M., \& Colome, C. 1994, ApJ, 433, 199

Lis, D. C., Menten, K. M., \& Zylka, R. 1999, ApJ, 527, 856

Liu, T., Zhang, H., Wu, Y., Qin, S.-L., \& Miller, M. 2011, ApJ, 734, 22

Lodato, G., \& Bertin, G. 2001, A\&A, 375, 455

Lodato, G., \& Bertin, G. 2003, A\&A, 408, 1015

Lombardi, M., Alves, J., \& Lada, C. J. 2011, A\&A, 535, A16

Looney, L. W., Mundy, L. G., \& Welch, W. J. 1997, ApJL, 484, L157

Lorenzetti, D., Giannini, T., \& Nisini, B. 2001, in The Promise of the Herschel Space Observatory, ed. G. L. Pilbratt, J. Cernicharo, A. M. Heras, T. Prusti, \& R. Harris (ESA SP-460; Noordwijk: ESA), 451

Lorenzetti, D., Giannini, T., Nisini, B., et al. 2000, A\&A, 357, 1035

Malbet, F., Lachaume, R., Berger, J.-P., et al. 2005, A\&A, 437, 627

Mattila, K., Liljeström, T., \& Toriseva, M. 1989, in ESO Workshop on Low Mass Star Formation and Pre-main Sequence Objects, ed. B. Reipurth (Garching: ESO), 153

McGehee, P. M., Smith, J. A., Henden, A. A., et al. 2004, ApJ, 616, 1058

McMuldroch, S., Blake, G. A., \& Sargent, A. I. 1995, AJ, 110, 354

McMuldroch, S., Sargent, A. I., \& Blake, G. A. 1993, AJ, 106, 2477

Mendoza, E. E. 1971, BOTT, 6, 137

Mendoza V., E. E. 1971, ApJL, 169, L117

Menten, K. M., Reid, M. J., Forbrich, J., \& Brunthaler, A. 2007, A\&A, 474, 515

Miller, A. A., Hillenbrand, L. A., Covey, K. R., et al. 2011, ApJ, 730, 80

Moneti, A., Forrest, W. J., Pipher, J. L., \& Woodward, C. E. 1988, ApJ, 327,870

Mookerjea, B., Ghosh, S. K., Karnik, A. D., et al. 1999, ApJ, 522, 285 
Moriarty-Schieven, G. H., Aspin, C., \& Davis, G. R. 2008, AJ, 136, 1658 Moriarty-Schieven, G. H., \& Snell, R. L. 1988, ApJ, 332, 364

Moriarty-Schieven, G. H., Wannier, P. G., Keene, J., \& Tamura, M. 1994, ApJ, 436, 800

Motte, F., \& André, P. 2001, A\&A, 365, 440

Munari, U., Milani, A., Valisa, P., \& Semkov, E. 2010, ATel, 2808, 1

Mundt, R., \& Eislöffel, J. 1998, AJ, 116, 860

Mundt, R., \& Fried, J. W. 1983, ApJL, 274, L83

Mundt, R., Stocke, J., Strom, S. E., Strom, K. M., \& Anderson, E. R. 1985, ApJL, 297, L41

Murakami, H., Baba, H., Barthel, P., et al. 2007, PASJ, 59, 369

Muzerolle, J., Megeath, S. T., Flaherty, K. M., et al. 2005, ApJL, 620, L107

Myers, P. C., Fuller, G. A., Mathieu, R. D., et al. 1987, ApJ, 319, 340

Neckel, T., \& Staude, H. J. 1984, A\&A, 131, 200

Odenwald, S. F. 1989, AJ, 97, 801

Osorio, M., D’Alessio, P., Muzerolle, J., Calvet, N., \& Hartmann, L. 2003, ApJ, 586,1148

Park, S., \& Kenyon, S. J. 2002, AJ, 123, 3370

Parsamian, E. S. 1965, IzArm, 18, 146

Peneva, S. P., Semkov, E. H., Munari, U., \& Birkle, K. 2010, A\&A, 515, A24

Pérez, L. M., Lamb, J. W., Woody, D. P., et al. 2010, ApJ, 724, 493

Persi, P., Marenzi, A. R., Kaas, A. A., et al. 1999, AJ, 117, 439

Persi, P., Tapia, M., Gòmez, M., et al. 2007, AJ, 133, 1690

Pfalzner, S. 2008, A\&A, 492, 735

Phillips, J. P., White, G. J., Ade, P. A. R., et al. 1982, A\&A, 116, 130

Poglitsch, A., Waelkens, C., Geis, N., et al. 2010, A\&A, 518, L2

Polomski, E. F., Woodward, C. E., Holmes, E. K., et al. 2005, AJ, 129, 1035

Popham, R., Kenyon, S., Hartmann, L., \& Narayan, R. 1996, ApJ, 473, 422

Prato, L., Lockhart, K. E., Johns-Krull, C. M., \& Rayner, J. T. 2009, AJ, 137,3931

Prusti, T., Bontekoe, Tj. R., Chiar, J. E., Kester, D. J. M., \& Whittet, D. C. B. 1993, A\&A, 279, 163

Quanz, S. P., Apai, D., \& Henning, T. 2007a, ApJ, 656, 287

Quanz, S. P., Henning, T., Bouwman, J., Ratzka, T., \& Leinert, C. 2006, ApJ, 648,472

Quanz, S. P., Henning, T., Bouwman, J., et al. 2007b, ApJ, 668, 359

Rebull, L. M., Guieu, S., Stauffer, J. R., et al. 2011, ApJS, 193, 25

Reipurth, B. 1985a, A\&A, 143, 435

Reipurth, B. 1985b, in ESO-IRAM-Onsala Workshop on (Sub)Millimeter Astronomy, ed. P. A. Shaver \& K. Kjar (Garching: ESO), 459

Reipurth, B., \& Aspin, C. 1997, AJ, 114, 2700

Reipurth, B., \& Aspin, C. 2004a, ApJL, 606, L119

Reipurth, B., \& Aspin, C. 2004b, ApJL, 608, L65

Reipurth, B., Aspin, C., Beck, T., et al. 2007, AJ, 133, 1000

Reipurth, B., Aspin, C., \& Herbig, G. H. 2012, ApJL, 748, L5

Reipurth, B., \& Bally, J. 1986, Natur, 320, 336

Reipurth, B., Bally, J., \& Devine, D. 1997, AJ, 114, 2708

Reipurth, B., Chini, R., Krugel, E., Kreysa, E., \& Sievers, A. 1993, A\&A, 273,221

Reipurth, B., Hartmann, L., Kenyon, S. J., Smette, A., \& Bouchet, P. 2002a, AJ, 124,2194

Reipurth, B., Rodríguez, L. F., Anglada, G., \& Bally, J. 2002b, AJ, 124, 1045

Rieke, G. H., Young, E. T., Engelbracht, C. W., et al. 2004, ApJS, 154, 25

Robitaille, T. P., Whitney, B. A., Indebetouw, R., \& Wood, K. 2007, ApJS, 169,328

Robitaille, T. P., Whitney, B. A., Indebetouw, R., Wood, K., \& Denzmore, P. 2006, ApJS, 167, 256

Rodríguez, L. F., D’Alessio, P., Wilner, D. J., et al. 1998, Natur, 395, 355

Rodríguez, L. F., Porras, A., Claussen, M. J., et al. 2003, ApJL, 586, L137

Rosolowsky, E., Dunham, M. K., Ginsburg, A., et al. 2010, ApJS, 188, 123

Roy, A., Ade, P. A. R., Bock, J. J., et al. 2011, ApJ, 730, 142

Sandell, G., \& Aspin, C. 1998, A\&A, 333, 1016
Sandell, G., \& Weintraub, D. A. 2001, ApJS, 134, 115

Sandstrom, K. M., Peek, J. E. G., Bower, G. C., Bolatto, A. D., \& Plambeck, R. L. 2007, ApJ, 667, 1161

Sato, S., Okita, K., Yamashita, T., et al. 1992, ApJ, 398, 273

Schütz, O., Meeus, G., \& Sterzik, M. F. 2005, A\&A, 431, 165

Semkov, E. H., \& Peneva, S. P. 2012, IBVS, 6025, 1

Semkov, E. H., Peneva, S. P., Munari, U., Milani, A., \& Valisa, P. 2010, A\&A, 523, L3

Semkov, E. H., Peneva, S. P., Munari, U., et al. 2012, A\&A, 542, A43

Siess, L., Dufour, E., \& Forestini, M. 2000, A\&A, 358, 593

Simon, T., Morrison, N. D., Wolff, S. C., \& Morrison, D. 1972, PASP, 84,644

Skrutskie, M. F., Cutri, R. M., Stiening, R., et al. 2006, AJ, 131, 1163

Smith, H. A., Thronson, H. A., Jr., Lada, C. J., et al. 1982, ApJ, 258, 170

Snell, R. L., Bally, J., Strom, S. E., \& Strom, K. M. 1985, ApJ, 290, 587

Snell, R. L., Dickman, R. L., \& Huang, Y. L. 1990, ApJ, 352, 139

Snell, R. L., Loren, R. B., \& Plambeck, R. L. 1980, ApJL, 239, L17

Stanke, Th., McCaughrean, M. J., \& Zinnecker, H. 2000, A\&A, 355, 639

Staude, H. J., \& Neckel, T. 1991, A\&A, 244, L13

Staude, H. J., \& Neckel, T. 1992, ApJ, 400, 556

Stocke, J. T., Hartigan, P. M., Strom, S. E., et al. 1988, ApJS, 68, 229

Straizys, V., Meistas, E., Vansevicius, V., \& Goldberg, E. P. 1989, A\&A, 222, 82

Strom, K. M., \& Strom, S. E. 1993, ApJL, 412, L63

Strom, K. M., Strom, S. E., \& Vrba, F. J. 1976, AJ, 81, 320

Szeifert, T., Hubrig, S., Schöller, M., et al. 2010, A\&A, 509, L7

Tapia, M., Persi, P., Bohigas, J., \& Ferrari-Toniolo, M. 1997, AJ, 113, 1769

Tapia, M., Persi, P., Bohigas, J., Roth, M., \& Gómez, M. 2006, MNRAS, 367,513

Terranegra, L., Chavarria-K., C., Diaz, S., \& Gonzalez-Patino, D. 1994, A\&AS, 104,557

Thiebaut, E., Bouvier, J., Blazit, A., et al. 1995, A\&A, 303, 795

Thommes, J., Reipurth, B., Aspin, C., \& Herbig, G. H. 2011, CBET, 2795, 1

Toomre, A. 1964, ApJ, 139, 1217

Tsukagoshi, T., Kitamura, Y., Kawabe, R., et al. 2005, in Protostars and Planets V, ed. B. Reipurth, D. Jewitt, \& K. Keil (Tucson, AZ: Univ. Arizona Press), 8475

Vacca, W. D., Cushing, M. C., \& Simon, T. 2004, ApJL, 609, L29

van den Ancker, M. E., Blondel, P. F. C., Tjin A Djie, H. R. E., et al. 2004, MNRAS, 349, 1516

Vorobyov, E. I., \& Basu, S. 2005, ApJL, 633, L137

Vorobyov, E. I., \& Basu, S. 2006, ApJ, 650, 956

Vorobyov, E. I., \& Basu, S. 2010, ApJ, 719, 1896

Walker, C. K., Adams, F. C., \& Lada, C. J. 1990, ApJ, 349, 515

Walter, F. M., Stringfellow, G. S., Sherry, W. H., \& Field-Pollatou, A. 2004, AJ, 128,1872

Weaver, W. B., \& Jones, G. 1992, ApJS, 78, 239

Weintraub, D. A., Sandell, G., \& Duncan, W. D. 1991, ApJ, 382, 270

Welin, G. 1971, IBVS, 581, 1

Welin, G. 1976, A\&A, 49, 145

Whelan, E. T., Dougados, C., Perrin, M. D., et al. 2010, ApJL, 720, L119

Whitney, B. A., Kenyon, S. J., \& Gomez, M. 1997, ApJ, 485, 703

Whitney, B. A., Wood, K., Bjorkman, J. E., \& Cohen, M. 2003a, ApJ, 598, 1079

Whitney, B. A., Wood, K., Bjorkman, J. E., \& Wolff, M. J. 2003b, ApJ, 591, 1049

Woody, D. P., Scott, S. L., Scoville, N. Z., et al. 1989, ApJL, 337, L41

Wright, E. L., Eisenhardt, P. R. M., Mainzer, A. K., et al. 2010, AJ, 140, 1868

Yang, J., Umemoto, T., Iwata, T., \& Fukui, Y. 1991, ApJ, 373, 137

Zhu, Z., Hartmann, L., Calvet, N., et al. 2008, ApJ, 684, 1281

Zhu, Z., Hartmann, L., Gammie, C., \& McKinney, J. C. 2009, ApJ, 701, 620

Zhu, Z., Hartmann, L., \& Gammie, C. 2010, ApJ, 713, 1143 